\title{
RECURSIONS FOR THE COMPUTATION OF MULTIPOLE TRANSLATION AND ROTATION COEFFICIENTS FOR THE 3-D HELMHOLTZ EQUATION
}

\author{
NAIL A. GUMEROV AND RAMANI DURAISWAMI *
}

\begin{abstract}
We develop exact expressions for the coef cients of series representations of translations and rotations of local and multipole fundamental solutions of the Helmholtz equation in spherical coordinates. These expressions are based on the derivation of recurrence relations, some of which, to our knowledge are presented here for the rst time. The symmetry and other properties of the coef cients are also examined, and based on these, ef cient procedures for calculating them are presented. Our expressions are direct, and do not use the Clebsch-Gordan coef cients or the Wigner 3- $j$ symbols, though we compare our results with methods that use these, to prove their accuracy. For evaluating a $N_{t}$ term truncation of the translated series (involving $O\left(N_{t}^{2}\right)$ multipoles), compared to previous exact expressions that require $O\left(N_{t}^{5}\right)$ operations, our expressions require $O\left(N_{t}^{3}\right)$ evaluations.
\end{abstract}

Key words. Helmholtz equation, multipole solutions, translation and rotation coef cients, fast evaluation.

AMS subject classi cations. 33C55, 33C10, 35J05, 65N38, 65N99, 65Y20

1. Introduction. In several scienti c computing applications, the solution to the Helmholtz or Maxwell Equations is expressed in terms of the singular (multipole) and regular solutions of the Helmholtz equation in spherical coordinates, centered at various points. Series of such solutions (see Eq. (2.16)) in one coordinate system must be expressed, in terms of series of singular or regular solutions in another coordinate system. Such expressions are guaranteed to exist by the completeness of the functions on a sphere. Addition theorems [5], [15] provide the expressions for the coef cients of the series in the shifted coordinates, in terms of the original coef cients. The paper by Epton and Dembart [8] provides an introduction to expressions of the coef cients. Chew [22] applied differentiation theorems for spherical functions similar to those in this paper, to obtain recursions for the translation coef cients.

One important scienti c computing area where there is a need for such expressions is in the Fast Multipole Method (FMM) solution of the Helmholtz and Maxwell equations [9, 27, 10]. The FMM algorithm was referred in [1] as one of the top algorithms of the 20th century. Here the complexity of the translation expressions on the one hand, and the numerical accuracy achievable on the other, are key barriers to use of these methods to more complicated problems that are of interest, and these are thus an area of active research. Other scienti c computing areas where there is a need for such translation theorems are in the solution of boundary value problems of scatterings from many spheres [24], and in the use of the T-matrix method for solution of scattering problems from many scatterers [14]. Note that in some multipole methods (e.g. [24]) computation of each entry of the translation matrix is needed. In this case the recursive computation of the matrix elements provides the algorithm with theoretical minimum of asymptotic complexity. For speci c applications we refer the reader to these papers.

In this paper we follow and extend the approach of [22] to develop general recursive methods for obtaining translation and rotation coef cients. We also derive recursions for a particular type of translation coef cients, which we call "coaxial" translation coef cients. An algorithm for fast and exact computation of the latter coef cients together with the rotation coef cients yields a translation algorithm based on a rotation-coaxial translation decomposition with the lower asymptotic complexity. While the relations are derived here for the Helmholtz equation with real $k$ in Equation (2.1) below, in fact they are applicable for arbitrary complex non-zero $k$, and appear in the modi ed Helmholtz equation describing screened coulombic ("Yukawa") interactions, or in the equation obtained on Fourier transform of the heat conduction equation, telegraph, or of the wave equation describing propagation of waves in media with relaxation, dispersion and dissipation.

For similar reasons, there is also a need for development of translation expressions for other equations of mathematical physics, such as the Laplace and linearized Poisson-Boltzmann equations. The approach we follow in deriving the translation is rather general, and might be useful in obtaining similar recursions for these other equations. Finally rotations on a sphere, and the spherical harmonics play important roles in many areas scienti c computation. In quantum chemistry, they occur for instance as factors of atomic orbitals and as factors in multipole expansions. The recursion that we develop for computation of the rotation coef cients is different from those used in this eld (see e.g., $[18,19])$. A computationally ef cient recursion for rotation coef cients which deals with real numbers is presented here.

* \{gumerov, ramani \}@umiacs . umd .edu Perceptual Interfaces and Reality Laboratory, Institute for Advanced Computer Studies, University of Maryland, College Park, MD 20742 
2. Background. We consider the Helmholtz equation in 3-D for the complex function $\psi(\mathbf{r})$, given by

$$
\nabla^{2} \psi+k^{2} \psi=0
$$

where $\nabla^{2}$ is the Laplace operator $\nabla \cdot(\nabla)$, and $k$ is a real scalar (the wavenumber). The transformation between spherical coordinates and Cartesian coordinates with a common origin $(x, y, z) \rightarrow(r, \theta, \varphi)$ is given by

$$
x=r \sin \theta \cos \varphi, \quad y=r \sin \theta \sin \varphi, \quad z=r \cos \theta .
$$

The gradient and Laplacian of a function $\psi$ in spherical coordinates are

$$
\begin{aligned}
\nabla \psi & =\mathbf{i}_{r} \frac{\partial \psi}{\partial r}+\mathbf{i}_{\theta} \frac{1}{r} \frac{\partial \psi}{\partial \theta}+\mathbf{i}_{\varphi} \frac{1}{r \sin \theta} \frac{\partial \psi}{\partial \varphi}, \\
\nabla \cdot(\nabla \psi) & =\nabla^{2} \psi=\frac{1}{r^{2}} \frac{\partial}{\partial r}\left(r^{2} \frac{\partial \psi}{\partial r}\right)+\frac{1}{r^{2} \sin \theta} \frac{\partial}{\partial \theta}\left(\sin \theta \frac{\partial \psi}{\partial \theta}\right)+\frac{1}{r^{2} \sin ^{2} \theta} \frac{\partial^{2} \psi}{\partial \varphi^{2}} .
\end{aligned}
$$

where $\left(\mathbf{i}_{r}, \mathbf{i}_{\theta}, \mathbf{i}_{\varphi}\right)$ is a right-handed orthonormal basis in spherical coordinates. Solutions of Helmholtz equation in spherical coordinates can be expressed in the factored form ("separation of variables")

$$
\psi_{n}^{m}(r, \theta, \varphi)=\Pi_{n}(r) \Theta_{n}^{m}(\theta) \Phi^{m}(\varphi),
$$

where the function $\Theta_{n}^{m}$ is periodic with period $\pi$ and $\Phi^{m}$ is periodic with period $2 \pi$. The spherical harmonics provide such a periodic basis

$$
\begin{aligned}
\Theta_{n}^{m}(\theta) \Phi^{m}(\varphi) & =Y_{n}^{m}(\theta, \varphi)=(-1)^{m} \sqrt{\frac{2 n+1}{4 \pi} \frac{(n-|m|) !}{(n+|m|) !}} P_{n}^{|m|}(\mu) e^{i m \varphi}, \quad \mu=\cos \theta, \\
n & =0,1,2, \ldots ; \quad m=-n, \ldots, n,
\end{aligned}
$$

where $P_{n}^{|m|}(\mu)$ are the associated Legendre functions [6]. The spherical harmonics are also sometimes called surface harmonics of the rst kind, tesseral for $m<n$ and sectorial for $m=n$. We will use the de nition of the associated Legendre function $P_{n}^{m}(\mu)$ that is consistent with the value on the cut $(-1,1)$ of the hypergeometric function $P_{n}^{m}(z)$ (see Abramowitz and Stegun, [6]). These functions can be obtained from the Legendre polynomials $P_{n}(\mu)$ via the Rodrigues' formula

$$
P_{n}^{m}(\mu)=(-1)^{m}\left(1-\mu^{2}\right)^{m / 2} \frac{d^{m}}{d \mu^{m}} P_{n}(\mu), \quad P_{n}(\mu)=\frac{1}{2^{n} n !} \frac{d^{n}}{d \mu^{n}}\left(\mu^{2}-1\right)^{n} .
$$

Our de nition of spherical harmonics coincides with that of Epton and Dembart [8], except for a factor $\sqrt{(2 n+1) / 4 \pi}$, which we include to make them an orthonormal basis over the sphere. As remarked in Ref. [8] the de nition of spherical harmonics has an important bearing on developing an ef cient multipole translation theory, and needs further research.

The spherical harmonics de ned by (2.5) form a complete orthonormal system on $L^{2}\left(S_{u}\right)$, where $S_{u}$ is the surface of the unit sphere $x^{2}+y^{2}+z^{2}=1$ :

$$
\left(Y_{n}^{m}, Y_{l}^{s}\right)=\int_{0}^{\pi} \sin \theta d \theta \int_{0}^{2 \pi} Y_{n}^{m}(\theta, \varphi) \bar{Y}_{l}^{s}(\theta, \varphi) d \varphi=\int_{0}^{\pi} \sin \theta d \theta \int_{0}^{2 \pi} Y_{n}^{m}(\theta, \varphi) Y_{l}^{-s}(\theta, \varphi) d \varphi=\delta_{n l} \delta_{m s},
$$

where $\delta_{n l}$ is the Kronecker delta. An arbitrary surface function $F(\theta, \varphi)$ can be expanded over this orthonormal basis as

$$
F(\theta, \varphi)=\sum_{n=0}^{\infty} \sum_{m=-n}^{n} F_{n}^{m} Y_{n}^{m}(\theta, \varphi)
$$

where the coef cients of the expansion $F_{n}^{m}$ are given by

$$
F_{n}^{m}=\left(F, Y_{n}^{m}\right)=\int_{0}^{\pi} \sin \theta d \theta \int_{0}^{2 \pi} F(\theta, \varphi) Y_{n}^{-m}(\theta, \varphi) d \varphi .
$$


The dependence of the function $\Pi_{n}$ on the radial coordinate, in Eq. (2.4), is described by

$$
\frac{d}{d r}\left(r^{2} \frac{d \Pi_{n}}{d r}\right)+\left[k^{2} r^{2}-n(n+1)\right] \Pi_{n}=0,
$$

which is the spherical Bessel equation. Particular solutions are the spherical Bessel functions of the rst and the second kind, $j_{n}$ and $y_{n}$, related respectively to the Bessel and Neumann functions of fractional order, $J_{n+1 / 2}$ and $Y_{n+1 / 2}$ :

$$
\Pi_{n}=j_{n}(k r)=\sqrt{\frac{\pi}{2 k r}} J_{n+1 / 2}(k r), \quad \Pi_{n}=y_{n}(k r)=\sqrt{\frac{\pi}{2 k r}} Y_{n+1 / 2}(k r) .
$$

When problems are posed on in nite domains, linear combinations of these solutions, called the spherical Hankel functions of the rst and the second kind, are used since they can be used to represent outgoing and incoming waves

$$
h_{n}^{(1)}(k r)=j_{n}(k r)+i y_{n}(k r), \quad h_{n}^{(2)}(k r)=j_{n}(k r)-i y_{n}(k r) .
$$

In the problems we are interested in, we will need functions which are either regular at the origin, or satisfy the Sommerfeld condition

$$
\lim _{r \rightarrow \infty} r\left(\frac{\partial \psi}{\partial r}-i k \psi\right)=0
$$

at in nity. These functions respectively are $j_{n}(k r)$ (regular as $r \rightarrow 0$,) and $h_{n}^{(1)}(k r)$ (outgoing waves). Accordingly, we can express the general solutions of Helmholtz equation in terms of either the 'elementary regular solutions' $R_{n}^{m}$ or the 'elementary singular solutions' $S_{n}^{m}$ de ned by

$$
R_{n}^{m}(\mathbf{r})=j_{n}(k r) Y_{n}^{m}(\theta, \varphi), \quad S_{n}^{m}(\mathbf{r})=h_{n}^{(1)}(k r) Y_{n}^{m}(\theta, \varphi), \quad n=0,1,2, \ldots ; \quad m=-n, \ldots, n,
$$

and which are linearly independent. The singular solution $S_{n}^{m}(\mathbf{r})$ sometimes is called as the multipole of order $m$ and degree $n$ centered at the origin (in some papers the multipoles are introduced as derivatives of the Green function). Since only the functions $h_{n}^{(1)}(k r)$ will be considered below, we will drop the superscript (1) for notational simplicity.

Because the functions $h_{n}(k r)$ and $j_{n}(k r)$ have similar recurrence properties, and when an expression applies to both types of functions, we will use the notation

$$
F_{n}^{m}(\mathbf{r})=f_{n}(k r) Y_{n}^{m}, \quad f=h, j ; \quad F=S, R .
$$

2.1. Reexpansions of Elementary Solutions. Solutions of the Helmholtz equation, $\psi$, in a nite or an in nite domain can be expressed in terms of the functions $S_{n}^{m}$ and $R_{n}^{m}$ and we can express it as (see e.g., [5])

$$
\psi(\mathbf{r})=\sum_{n=0}^{\infty} \sum_{m=-n}^{n}\left[A_{n}^{m} S_{n}^{m}(\mathbf{r})+B_{n}^{m} R_{n}^{m}(\mathbf{r})\right],
$$

where $A_{n}^{m}$ and $B_{n}^{m}$ are expansion coef cients. In particular, for $A_{n}^{m}=0$ such a series describe any regular solution inside a sphere, and for $B_{n}^{m}=0$ any radiating solution in the space exterior to a sphere circumscribing a scatterer, and for $A_{n}^{m} \neq 0$ and $B_{n}^{m} \neq 0$ any regular solution in a spherical layer. These series also can be considered to be centered at different locations, and sums of such functions provide solutions to the Helmholtz equation in multiply connected domains, such as appear in multiple scattering problems, or in domains with complex boundaries. In these cases, the series centered at different locations should be "translated" to provide local or far eld expansions, and the coef cients of the translated series should be evaluated. These coef cients could be obtained by taking appropriate scalar products, resulting in integral expressions for the coef cients, which, absent analytical expressions, must be evaluated numerically via quadrature, and are thus inef cient. "Translation theorems" provide explicit ways for evaluating the coef cients, and are thus more ef cient. The translation theorem expression for the monopole source centered at the origin is well known and given in many textbooks (see e.g., [5]). The monopole can be expanded in a series of spherical harmonics centered about a point $q$ using the following identity

$$
G(\mathbf{r})=\frac{e^{i k|\mathbf{r}|}}{4 \pi|\mathbf{r}|}=i k \sum_{n=0}^{\infty} \sum_{m=-n}^{n} R_{n}^{-m}\left(-\mathbf{r}_{q}^{\prime}\right) S_{n}^{m}\left(\mathbf{r}_{q}\right), \quad\left|\mathbf{r}_{q}^{\prime}\right| \leqslant\left|\mathbf{r}_{q}\right|,
$$




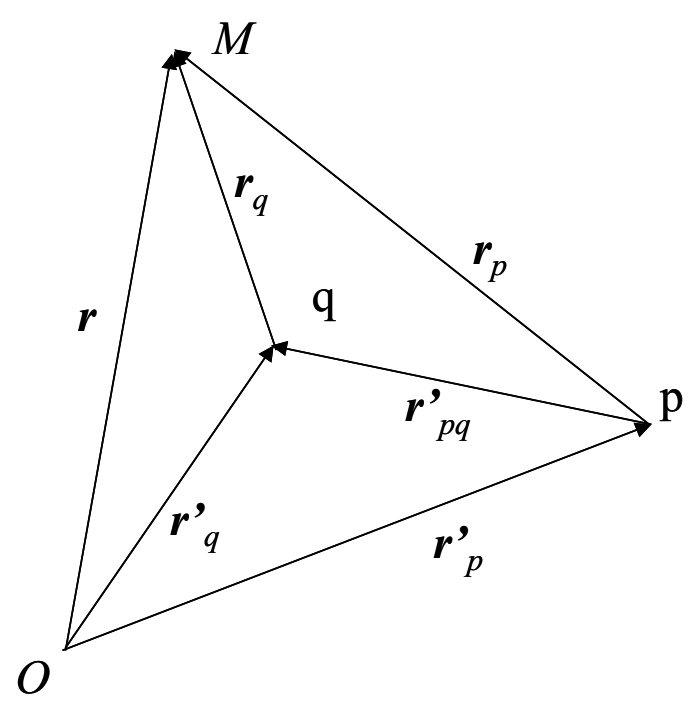

FIG. 2.1. Coordinates of points in various reference frames.

where $\mathbf{r}_{q}$ is the radius-vector of the eld point in the reference frame centered at $q$ and $\mathbf{r}_{q}^{\prime}$ is the radius-vector of the point $q$ in the original reference frame. Thus in this expression the multipole of order zero, centered at the origin has been translated to a series of multipoles centered at a point $q$, and the series has coef cients $i k R_{n}^{-m}\left(-\mathbf{r}_{q}^{\prime}\right)$. If the outer summation of the series (2.17) is truncated at $n=N_{t}$, the series has $O\left(N_{t}+1\right)^{2}$ terms, and the monopole can be translated to a new location in $O\left(N_{t}^{2}\right)$ operations.

Such succinct expressions are usually not available for higher order multipoles. Exact expressions for multipole translations for the Helmholtz equation have been presented in [8]. However these expressions are relatively cumbersome as they use the Wigner or Clebsch-Gordan coef cients, and are relatively expensive to compute, requiring $O\left(N_{t}^{5}\right)$ operations to evaluate the $O\left(N_{t}^{2}\right)$ terms.

2.2. Translations. We wish to represent $S_{n}^{m}\left(\mathbf{r}_{p}\right)$ and $R_{n}^{m}\left(\mathbf{r}_{p}\right)$ as sums of singular or regular elementary solutions with the center of expansion speci ed at some other point $\mathbf{r}=\mathbf{r}_{q}^{\prime}$. To obtain such representations we introduce spherical coordinates centered at $\mathbf{r}=\mathbf{r}_{q}^{\prime}$, so $\mathbf{r}-\mathbf{r}_{q}^{\prime}=\mathbf{r}_{q}=\left(r_{q}, \theta_{q}, \varphi_{q}\right)$. By de nition, we have

$$
\mathbf{r}=\mathbf{r}_{p}+\mathbf{r}_{p}^{\prime}=\mathbf{r}_{q}+\mathbf{r}_{q}^{\prime}, \quad \mathbf{r}_{p}=\mathbf{r}_{q}+\mathbf{r}_{p q}^{\prime}, \quad \mathbf{r}_{p q}^{\prime}=\mathbf{r}_{q}^{\prime}-\mathbf{r}_{p}^{\prime}=\mathbf{r}_{p}-\mathbf{r}_{q},
$$

where the vector $\mathbf{r}_{p q}^{\prime}$ is directed from point $p$ to point $q$. This vector determines the radius of reexpansion $r_{p q}^{\prime}=\left|\mathbf{r}_{p q}^{\prime}\right|$. Inside the sphere with radius $r_{p q}^{\prime}$, centered at $\mathbf{r}=\mathbf{r}_{q}^{\prime}$, the solution is regular and can be represented as

$$
S_{n}^{m}\left(\mathbf{r}_{p}\right)=\sum_{l=0}^{\infty} \sum_{s=-l}^{l}(S \mid R)_{l n}^{s m}\left(\mathbf{r}_{p q}^{\prime}\right) R_{l}^{s}\left(\mathbf{r}_{q}\right), \quad\left|\mathbf{r}_{q}\right|<\left|\mathbf{r}_{p q}^{\prime}\right|, \quad p \neq q .
$$

The singular elementary solution outside this sphere satis es the radiation conditions, and therefore we can represent $S_{n}^{m}$ in a series of multipole solutions:

$$
S_{n}^{m}\left(\mathbf{r}_{p}\right)=\sum_{l=0}^{\infty} \sum_{s=-l}^{l}(S \mid S)_{l n}^{s m}\left(\mathbf{r}_{p q}^{\prime}\right) S_{l}^{s}\left(\mathbf{r}_{q}\right), \quad\left|\mathbf{r}_{q}\right|>\left|\mathbf{r}_{p q}^{\prime}\right| .
$$

The regular elementary solutions inside a nite domain can be reexpanded in a series of regular elementary solutions near an arbitrary point, so that

$$
R_{n}^{m}\left(\mathbf{r}_{p}\right)=\sum_{l=0}^{\infty} \sum_{s=-l}^{l}(R \mid R)_{l n}^{s m}\left(\mathbf{r}_{p q}^{\prime}\right) R_{l}^{s}\left(\mathbf{r}_{q}\right) .
$$




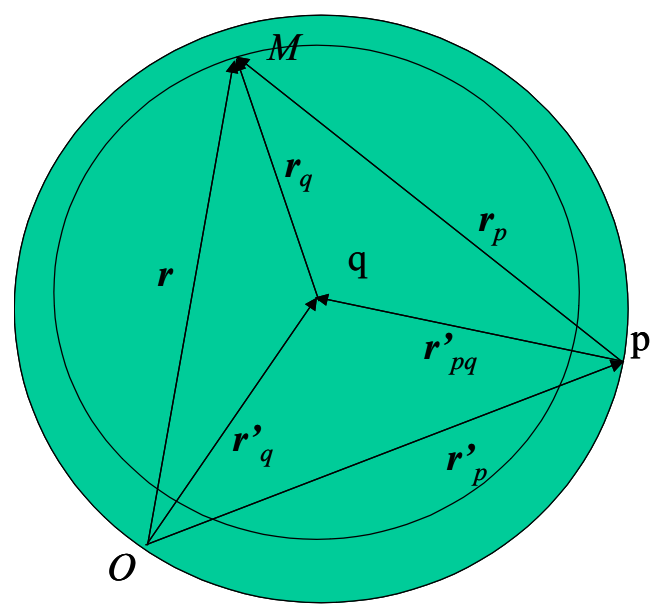

FIG. 2.2. Illustration of the reexpansion of the singular to regular solution (2.19). Such reexpansion can be performed inside the dark sphere. It can be used for a eld point $M$, since the distance to this point from point $q$ is smaller than the radius of the dark sphere.

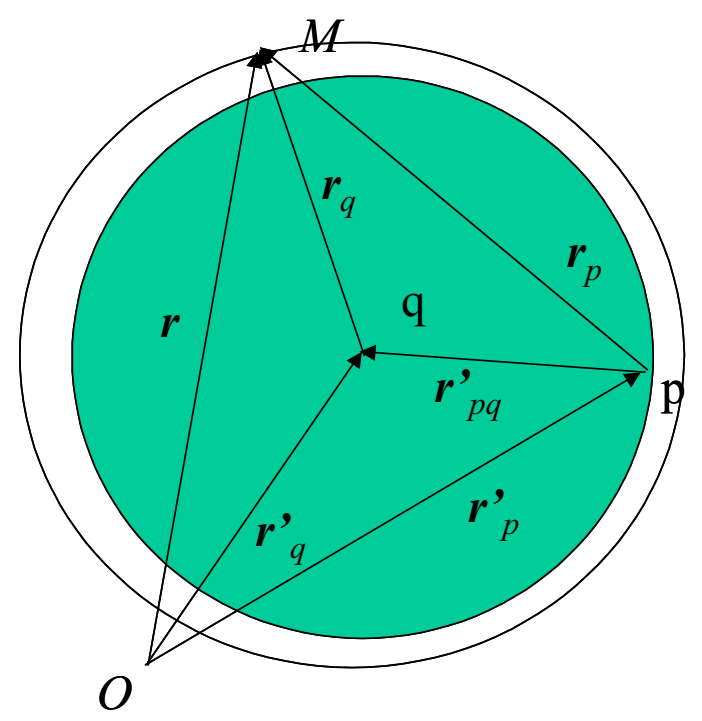

FIG. 2.3. Illustration of the reexpansion of the singular to singular solution (2.20). Such reexpansion can be performed outside the dark sphere. It can be used for a eld point $M$, since the distance to this point from point $q$ is larger than the radius of the dark sphere.

In expansions (2.19)-(2.21) the symbols $(S \mid R),(S \mid S)$ and $(R \mid R)$ denote that the singular $(S)$ and regular $(R)$ elementary solutions are reexpanded in series of regular or singular elementary solutions, respectively, with coef cients of reexpansion $(S \mid R)_{l n}^{s m},(S \mid S)_{l n}^{s m}$ and $(R \mid R)_{l n}^{s m}$.

Note that we do not consider the reexpansion of regular solutions in terms multipoles, i.e. $(R \mid S)$. If such a reexpansion were possible, then the regular solution would be "radiating" at in nity, which cannot be true. Therefore such reexpansions cannot be used either for in nite domains, or for nite domains including the singular point of the center of the expansion.

With such translations of the basis functions the coef cients in sums of type (2.16) can be also translated by multiplication of corresponding translation matrices by the vectors of coef cients. For example, if we have a far- eld expansion of a radiating function $\mathcal{F}\left(\mathbf{r}_{p}\right)$,

$$
\psi\left(\mathbf{r}_{p}\right)=\sum_{n=0}^{\infty} \sum_{m=-n}^{n} A_{n}^{m} S_{n}^{m}\left(\mathbf{r}_{p}\right)
$$


it can be converted to a local expansion near some point $q$ with the aid of matrix $(R \mid R)_{l n}^{s m}$ :

$$
\psi\left(\mathbf{r}_{p}\right)=\sum_{l=0}^{\infty} \sum_{s=-l}^{l} C_{l}^{s}\left(\mathbf{r}_{p q}^{\prime}\right) R_{l}^{s}\left(\mathbf{r}_{q}\right), \quad C_{l}^{s}\left(\mathbf{r}_{p q}^{\prime}\right)=\sum_{n=0}^{\infty} \sum_{m=-n}^{n}(S \mid R)_{l n}^{s m}\left(\mathbf{r}_{p q}^{\prime}\right) A_{n}^{m}, \quad\left|\mathbf{r}_{q}\right|<\left|\mathbf{r}_{p q}^{\prime}\right| .
$$

2.3. Rotations. We also consider transforms of multipole expansions due to rotation of one Cartesian system to another. Fast algorithms for computation of expansion coef cients of singular and regular solutions are needed for applications in quantum mechanics and, as will be seen, for fast computation of translation coef cients.

Let $\left(\mathbf{i}_{x}, \mathbf{i}_{y}, \mathbf{i}_{z}\right)$ and $\left(\mathbf{i}_{\widehat{x}}, \mathbf{i}_{\widehat{y}}, \mathbf{i}_{\bar{z}}\right)$ be two Cartesian systems of coordinates with a common origin. Let $Q$ be the rotation matrix that takes vector coordinates $\mathbf{a}$ in the rst coordinate system to the vector coordinates $\hat{\mathbf{a}}$ in the second coordinate system, so that

$$
\hat{\mathbf{a}}=Q \mathbf{a} \quad \text { with } \quad Q=\left[\begin{array}{lll}
\mathbf{i}_{\widehat{x}} \cdot \mathbf{i}_{x} & \mathbf{i}_{\widehat{x}} \cdot \mathbf{i}_{y} & \mathbf{i}_{\widehat{x}} \cdot \mathbf{i}_{z} \\
\mathbf{i}_{\widehat{y}} \cdot \mathbf{i}_{x} & \mathbf{i}_{\widehat{y}} \cdot \mathbf{i}_{y} & \mathbf{i}_{\widehat{y}} \cdot \mathbf{i}_{z} \\
\mathbf{i}_{\widehat{z}} \cdot \mathbf{i}_{x} & \mathbf{i}_{\widehat{z}} \cdot \mathbf{i}_{y} & \mathbf{i}_{\widehat{z}} \cdot \mathbf{i}_{z}
\end{array}\right] .
$$

We have the following transform of the spherical harmonics due to rotation of coordinates (e.g., see Wigner [17])

$$
Y_{n}^{m}(\theta, \varphi)=\sum_{\nu=-n}^{n} T_{n}^{\nu m}(Q) Y_{n}^{\nu}(\widehat{\theta}, \widehat{\varphi})
$$

where $T_{n}^{\nu m}(Q)$ are coef cients depending on the rotation matrix $Q$. Due to the de nition of the singular and regular solutions (2.14), and due to the fact that the magnitude of the vector does not change with rotation of coordinates, we can write the expansion for the multipole in the rotated coordinate system as

$$
S_{n}^{m}\left(\mathbf{r}_{p}\right)=\sum_{\nu=-n}^{n} T_{n}^{\nu m}(Q) S_{n}^{\nu}\left(\widehat{\mathbf{r}}_{p}\right), \quad R_{n}^{m}\left(\mathbf{r}_{p}\right)=\sum_{\nu=-n}^{n} T_{n}^{\nu m}(Q) R_{n}^{\nu}\left(\widehat{\mathbf{r}}_{p}\right), \quad\left|\widehat{\mathbf{r}}_{p}\right|=\left|\mathbf{r}_{p}\right|,
$$

where $\widehat{\mathbf{r}}_{p}$ is the denotes the point in the rotated coordinate system.

Using the reexpansions (e.g. equation (2.19)) the sum (2.22) can be represented in the form

$$
\psi\left(\mathbf{r}_{p}\right)=\sum_{n=0}^{\infty} \sum_{\nu=-n}^{n} C_{n}^{\nu} S_{n}^{\nu}\left(\widehat{\mathbf{r}}_{p}\right), \quad C_{n}^{\nu}=\sum_{m=-n}^{n} T_{n}^{\nu m}(Q) A_{n}^{m} .
$$

2.4. Complexity of Translations and Rotations. Usually we deal with expansions of singular or regular solutions $\psi(\mathbf{r})$ (such as Eq. (2.22) or Eq. (2.16)) with $B_{n}^{m}=0$ or $A_{n}^{m}=0$ ), where the outer sum is truncated at $n=N_{t}$. In this case the goal of the translation and rotation operations is to transform one set of $N_{t}^{2}$ coef cients representing $\psi(\mathbf{r})$ multiplying the regular or singular functions to another set of $N_{t}^{2}$ coef cients multiplying the regular or singular functions centered at another location, which represent the same function $\psi(\mathbf{r})$. A plausible way of doing this would be to look for relations between the $N_{t}^{2}$ coef cients. A general linear transform, would employ a relation that involved the action of a $N_{t}^{2} \times N_{t}^{2}$ matrix on the original coef cients to produce the new coef cients (see Eq. (2.23)). If the matrix were to be fully populated, it would contain $O\left(N_{t}^{4}\right)$ elements, and its evaluation would take $O\left(N_{t}^{4} \times \operatorname{cost}\right.$ of evaluating an element) operations, while direct evaluation of the matrix-vector product for the coef cients would require $O\left(N_{t}^{4}\right)$ operations. In many application areas $N_{t} \simeq 10^{2}$ or larger, and this expense can be signi cant.

The rst set of relations for obtaining the translation coef cients use the so-called Wigner symbols, and are well summarized in [8]. These expressions require $O\left(N_{t}^{5}\right)$ operations to evaluate the transformation matrix and $O\left(N_{t}^{4}\right)$ operations to perform the multiplication directly. Clearly, these are too expensive to evaluate for large $N_{t}$, especially when the computations must be done many times, as in the FMM, and where some error bounds suggest that the number of terms depends on the discretization. Since there are $O\left(N_{t}^{2}\right)$ coef cients, the minimum number of computations necessary to do the translations is $O\left(N_{t}^{2}\right)$.

To achieve faster methods for evaluation, research has progressed along two directions. A rst approach, and that which is quite common in the FMM literature, nesses the transformation problem. In this approach, an analytical "Fourier" transform on the sphere is performed, and the transform integral is evaluated using numerical quadrature. Multilevel fast multipole methods are implemented by referring to the transformed version of the multipole expansions. 
Details of this approach can be obtained from [9, 23, 21]. This approach based on so called "diagonal" forms of translation operators can achieve the translations necessary in a combination of several $O\left(N_{t}^{2}\right)$ operations with some $O\left(N_{t}^{3}\right)$ operations required for computation of surface integrals of the spherical transforms, which can be reduced to $O\left(N_{t}^{2} \log ^{2} N_{t}\right)$ using fast spherical transforms.

However, we need to make some remarks about these translation methods used in the FMM. First, the numerical quadrature of oscillatory functions that must be performed on the sphere may introduce large multipliers to the order estimates. For a practical problem, the number of computations performed by an $O\left(N_{t}^{3}\right)$ method with a smaller asymptotic multiplier can be less than that performed by, say, a $O\left(N_{t}^{2} \log ^{2} N_{t}\right)$ method with a larger asymptotic multiplier.

Second, estimates of the complexity of the FMM are usually presented as $O(P N)$ where $P$ is the single translation cost, and $N$ is the number of points for which FMM is used. A recent detailed look at the FMM algorithm [25], shows that it can be optimized by using better data structures, particularly by selection of a so-called "clustering" parameter. In this case the complexity of the method is $O\left(P^{1 / 2} N+N_{t}^{2} N\right)$. These estimates show that all translation algorithms with asymptotic complexity $P=O\left(N_{t}^{4}\right)$ or below result in an effective complexity of $O\left(N_{t}^{2} N\right)$ or $O\left(N_{t}^{2} N \log N\right)$ for the FMM. In this sense even a $O\left(N_{t}^{4}\right)$ "slow" translation algorithm deserves consideration, and $P=O\left(N_{t}^{3}\right)$ methods can be competitive with, say $O\left(N_{t}^{2} \log ^{2} N_{t}\right)$, methods (of course, any fast method providing the same accuracy is better than a slow method).

Finally, we note that at low frequencies the translation methods based on integral representations experience some problems, and there are publications dedicated to improvement of convergence of these methods [13]. The matrix based method of translation is applicable at suf ciently low frequencies (e.g., we tested the accuracy of solutions for $k^{\sim} 10^{-3}$, and characteristic distances of order 1 and obtained good results [26]).

3. Differentiation of Elementary Solutions. To derive recurrence relations for computation of the reexpansion coef cients we rst present some simple relations that arise from the differentiation of the elementary solutions. Let us de ne the following differential operators in spherical coordinates:

$$
\begin{aligned}
\partial_{z} & \equiv \frac{\partial}{\partial z}=\mu \frac{\partial}{\partial r}+\frac{1-\mu^{2}}{r} \frac{\partial}{\partial \mu}, \quad \mu=\cos \theta \\
\partial_{x y} & \equiv \frac{\partial}{\partial x}+i \frac{\partial}{\partial y}=\frac{e^{i \varphi}}{r \sqrt{1-\mu^{2}}}\left[\left(1-\mu^{2}\right)\left(r \frac{\partial}{\partial r}-\mu \frac{\partial}{\partial \mu}\right)+i \frac{\partial}{\partial \varphi}\right], \\
\overline{\partial_{x y}} & \equiv \frac{\partial}{\partial x}-i \frac{\partial}{\partial y}=\frac{e^{-i \varphi}}{r \sqrt{1-\mu^{2}}}\left[\left(1-\mu^{2}\right)\left(r \frac{\partial}{\partial r}-\mu \frac{\partial}{\partial \mu}\right)-i \frac{\partial}{\partial \varphi}\right], \\
\nabla & \equiv \mathbf{i}_{x} \frac{\partial}{\partial x}+\mathbf{i}_{y} \frac{\partial}{\partial y}+\mathbf{i}_{z} \frac{\partial}{\partial z}=\mathbf{i}_{x} \frac{1}{2}\left(\partial_{x y}+\overline{\partial_{x y}}\right)-\mathbf{i}_{y} \frac{i}{2}\left(\partial_{x y}-\overline{\partial_{x y}}\right)+\mathbf{i}_{z} \partial_{z} \\
& =\frac{1}{2}\left(\mathbf{i}_{x}-i \mathbf{i}_{y}\right) \partial_{x y}+\frac{1}{2}\left(\mathbf{i}_{x}+i \mathbf{i}_{y}\right) \overline{\partial_{x y}}+\mathbf{i}_{z} \partial_{z},
\end{aligned}
$$

Applying these operators we obtain the following theorems for the differentiation of the functions $S_{n}^{m}(\mathbf{r})$ or $R_{n}^{m}(\mathbf{r})$. The proofs of these theorems are based on the properties of the associated Legendre functions and spherical Hankel and Bessel functions and can be found in detail in the report [2], where numerical examples are provided as well. These theorems also can be found in [22].

THEOREM 3.1. For $k \neq 0$ and integer $n$ and $m$

$$
\frac{1}{k} \partial_{z} F_{n}^{m}(\mathbf{r})=a_{n-1}^{m} F_{n-1}^{m}(\mathbf{r})-a_{n}^{m} F_{n+1}^{m}(\mathbf{r}), \quad F=S, R .
$$

where

$$
a_{n}^{m}=0, \quad \text { for } n<|m| \quad a_{n}^{m}=a_{n}^{|m|}=\sqrt{\frac{(n+1+|m|)(n+1-|m|)}{(2 n+1)(2 n+3)}}, \text { for } n \geqslant|m| .
$$

THEOREM 3.2. For $k \neq 0$ and integer $m$ and $n$ :

$$
\frac{1}{k} \partial_{x y} F_{n}^{m}(\mathbf{r})=b_{n+1}^{-m-1} F_{n+1}^{m+1}(\mathbf{r})-b_{n}^{m} F_{n-1}^{m+1}(\mathbf{r}), \quad F=S, R .
$$


where

$b_{n}^{m}=\sqrt{\frac{(n-m-1)(n-m)}{(2 n-1)(2 n+1)}}$ for $0 \leqslant m \leqslant n ; \quad-\sqrt{\frac{(n-m-1)(n-m)}{(2 n-1)(2 n+1)}}$ for $-n \leqslant m<0 ; \quad 0$ for $|m|>n$,

THEOREM 3.3. For $k \neq 0$ and integer $n$ and $m$

$$
\frac{1}{k} \overline{\partial_{x y}} F_{n}^{m}(\mathbf{r})=b_{n+1}^{m-1} F_{n+1}^{m-1}(\mathbf{r})-b_{n}^{-m} F_{n-1}^{m-1}(\mathbf{r}), \quad F=S, R .
$$

where the coef cients $b_{n}^{m}$ are as de ned in Equation (3.5).

THEOREM 3.4. For $k \neq 0$ and integer $n$ and $m$

$$
\begin{aligned}
\frac{1}{k} \nabla F_{n}^{m}(\mathbf{r}) & =\frac{1}{2}\left(\mathbf{i}_{x}-i \mathbf{i}_{y}\right)\left[b_{n+1}^{-m-1} F_{n+1}^{m+1}(\mathbf{r})-b_{n}^{m} F_{n-1}^{m+1}(\mathbf{r})\right]+\frac{1}{2}\left(\mathbf{i}_{x}+i \mathbf{i}_{y}\right)\left[b_{n+1}^{m-1} F_{n+1}^{m-1}(\mathbf{r})-b_{n}^{-m} F_{n-1}^{m-1}(\mathbf{r})\right] \\
& +\mathbf{i}_{z}\left[a_{n-1}^{m} F_{n-1}^{m}(\mathbf{r})-a_{n}^{m} F_{n+1}^{m}(\mathbf{r})\right], \quad F=S, R .
\end{aligned}
$$

where the coef cients $a_{n}^{m}$ and $b_{n}^{m}$ are de ned by (3.3) and (3.5).

\section{Translation Coef cients.}

4.1. Integral Representation of Translation Coef cients. Before considering the ef cient evaluation of the translation coef cients $(S \mid R)_{l n}^{s m}\left(\mathbf{r}_{p q}^{\prime}\right)$ etc., we note their integral representations which immediately follow from de nitions (2.14), (2.19), and orthonormality of spherical harmonics (2.5):

$$
\begin{aligned}
(S \mid R)_{l n}^{s m}\left(\mathbf{r}_{p q}^{\prime}\right) & =\frac{1}{j_{l}\left(k r_{q}\right)}\left(S_{n}^{m}\left(\mathbf{r}_{p}\right), Y_{l}^{s}\left(\theta_{q}, \varphi_{q}\right)\right) \\
& =\frac{1}{j_{l}\left(k r_{q}\right)} \int_{-\pi}^{\pi} d \varphi_{q} \int_{0}^{\pi} h_{n}\left(k r_{p}\right) Y_{n}^{m}\left(\theta_{p}, \varphi_{p}\right) Y_{l}^{-s}\left(\theta_{q}, \varphi_{q}\right) \sin \theta_{q} d \theta_{q}, \quad r_{q}<\left|\mathbf{r}_{p q}^{\prime}\right|,
\end{aligned}
$$

Similarly, for the other reexpansion coef cients we have expressions

$$
\begin{aligned}
(R \mid R)_{l n}^{s m}\left(\mathbf{r}_{p q}^{\prime}\right) & =\frac{1}{j_{l}\left(k r_{q}\right)} \int_{-\pi}^{\pi} d \varphi_{q} \int_{0}^{\pi} j_{n}\left(k r_{p}\right) Y_{n}^{m}\left(\theta_{p}, \varphi_{p}\right) Y_{l}^{-s}\left(\theta_{q}, \varphi_{q}\right) \sin \theta_{q} d \theta_{q}, \\
(S \mid S)_{l n}^{s m}\left(\mathbf{r}_{p q}^{\prime}\right) & =\frac{1}{h_{l}\left(k r_{q}\right)} \int_{-\pi}^{\pi} d \varphi_{q} \int_{0}^{\pi} h_{n}\left(k r_{p}\right) Y_{n}^{m}\left(\theta_{p}, \varphi_{p}\right) Y_{l}^{-s}\left(\theta_{q}, \varphi_{q}\right) \sin \theta_{q} d \theta_{q}, \quad r_{q}>\left|\mathbf{r}_{p q}^{\prime}\right| .
\end{aligned}
$$

4.2. Structure of the Translation Coef cients. While the above integral representation provides an explicit way to calculate the reexpansion coef cients, this approach is not practical as the integral must be evaluated numerically, making the method computationally expensive. Moreover, the representations (4.1), (4.2), and (4.3) use coordinates of both the source point $\mathbf{p}$ and the target point $\mathbf{q}$, they are not useful for fast multipole methods. To be useful they would need to be rewritten in terms of the translation vector $\mathbf{r}_{p q}^{\prime}$ alone.

According to (2.18) and (2.19) we have

$$
S_{n}^{m}\left(\mathbf{r}_{q}+\mathbf{r}_{p q}^{\prime}\right)=\sum_{l=0}^{\infty} \sum_{s=-l}^{l}(S \mid R)_{l n}^{s m}\left(\mathbf{r}_{p q}^{\prime}\right) R_{l}^{s}\left(\mathbf{r}_{q}\right) .
$$

The function $S_{n}^{m}\left(\mathbf{r}_{q}+\mathbf{r}_{p q}^{\prime}\right)$ is regular inside $\left|\mathbf{r}_{q}\right| \leqslant\left|\mathbf{r}_{p q}^{\prime}\right|$ and satis es the Helmholtz equation:

$$
\left(\nabla^{2}+k^{2}\right) S_{n}^{m}\left(\mathbf{r}_{q}+\mathbf{r}_{p q}^{\prime}\right)=0
$$

The Laplace operator here can be considered to be acting either at $\quad$ xed $\mathbf{r}_{p q}^{\prime}$ or at $x e d \mathbf{r}_{q}$. In the former case we have

$$
\left(\nabla^{2}+k^{2}\right) R_{l}^{s}\left(\mathbf{r}_{q}\right)=0
$$


which also follows from the de nition of $R_{l}^{s}$. In the latter case we have

$$
\left(\nabla^{2}+k^{2}\right)(S \mid R)_{l n}^{s m}\left(\mathbf{r}_{p q}^{\prime}\right)=0 .
$$

The solution of this equation for the coef cients can be sought in the form of a multipole expansion as

$$
(S \mid R)_{l n}^{s m}\left(\mathbf{r}_{p q}^{\prime}\right)=\sum_{\alpha=0}^{\infty} \sum_{\beta=-\alpha}^{\alpha}(s \mid r)_{\alpha l n}^{\beta s m} S_{\alpha}^{\beta}\left(\mathbf{r}_{p q}^{\prime}\right)
$$

Indeed, in the expansion we need to only use the functions $S_{\alpha}^{\beta}\left(\mathbf{r}_{p q}^{\prime}\right)$, not $R_{\alpha}^{\beta}\left(\mathbf{r}_{p q}^{\prime}\right)$ or combinations of $R_{\alpha}^{\beta}\left(\mathbf{r}_{p q}^{\prime}\right)$ and $S_{\alpha}^{\beta}\left(\mathbf{r}_{p q}^{\prime}\right)$, since as $\left|\mathbf{r}_{p q}^{\prime}\right| \rightarrow \infty$, the solution should satisfy the Sommerfeld radiation conditions (see (4.4)). The coef cients $(s \mid r)_{\alpha l n}^{\beta s m}$ are purely numerical and do not depend on the locations of the multipole or the center of expansion.

Note that these coef cients can be related to the Clebsch-Gordan coef cients due to the addition theorem for the scalar wave functions [15], or to the Wigner 3- $j$ symbols [17], which are a more symmetrical form. The ClebschGordan coef cients $\left(j_{1} j_{2} m_{1} m_{2} \mid j_{1} j_{2} j m\right)$ are given by [6]:

$$
\left(j_{1} j_{2} m_{1} m_{2} \mid j_{1} j_{2} j m\right)=(-1)^{-j_{1}+j_{2}-m}(2 j+1)^{1 / 2}\left(\begin{array}{ccc}
j_{1} & j_{2} & j \\
m_{1} & m_{2} & -m
\end{array}\right),
$$

where the $2 \times 3$ matrix notation on the right hand side is used for the Wigner 3- $j$ symbols. In the paper of Epton and Dembart [8] the following expression (rewritten in the present notation) for the reexpansion coef cients is provided

$$
(S \mid R)_{l n}^{s m}\left(\mathbf{r}_{p q}^{\prime}\right)=\sum_{\alpha=0}^{\infty} \sum_{\beta=-\alpha}^{\alpha}\left[\frac{(2 n+1)(2 l+1)(2 \alpha+1)}{4 \pi}\right]^{1 / 2} i^{l-n+\alpha} E\left(\begin{array}{ccc}
m & -s & -\beta \\
n & l & \alpha
\end{array}\right) S_{\alpha}^{\beta}\left(\mathbf{r}_{p q}^{\prime}\right),
$$

where the symbol $E$ is de ned as

$E\left(\begin{array}{ccc}m & -s & -\beta \\ n & l & \alpha\end{array}\right)=4 \pi\left[\frac{4 \pi}{(2 n+1)(2 l+1)(2 \alpha+1)}\right]^{1 / 2} \int_{-\pi}^{\pi} d \varphi \int_{0}^{\pi} Y_{n}^{m}(\theta, \varphi) Y_{l}^{-s}(\theta, \varphi) Y_{\alpha}^{-\beta}(\theta, \varphi) \sin \theta d \theta$,

and is related to the Wigner $3-j$ symbols:

$$
E\left(\begin{array}{ccc}
m & -s & -\beta \\
n & l & \alpha
\end{array}\right)=4 \pi \epsilon_{m} \epsilon_{-s} \epsilon_{-\beta}\left(\begin{array}{ccc}
n & l & \alpha \\
0 & 0 & 0
\end{array}\right)\left(\begin{array}{ccc}
n & l & \alpha \\
m & -s & -\beta
\end{array}\right), \mathrm{w} \epsilon_{m}=\left\{\begin{array}{c}
(-1)^{m}, \quad m \geqslant 0 \\
1, \quad m \leqslant 0
\end{array} .\right.
$$

Computation of the Wigner 3- $j$ symbols, or $E$ symbols requires summations over several indices, and is computationally inef cient. We will not consider this way of obtaining the reexpansion coef cients and refer the reader to [8] for details of their computation.

Comparing (4.10) with (4.8), we note that

$$
(s \mid r)_{\alpha l n}^{\beta s m}=\left[\frac{(2 n+1)(2 l+1)(2 \alpha+1)}{4 \pi}\right]^{1 / 2} \quad i^{l-n+\alpha} E\left(\begin{array}{ccc}
m & -s & -\beta \\
n & l & \alpha
\end{array}\right) .
$$

The above $E$-symbol has a multiplier $\delta_{\beta, m-s}$, which means that

$$
(s \mid r)_{\alpha l n}^{\beta s m}=0, \text { for } \beta \neq m-s .
$$

It is also noteworthy that from the de nition (4.11) and orthonormality of the spherical harmonics we have

$$
E\left(\begin{array}{ccc}
0 & m & s \\
0 & n & l
\end{array}\right)=E\left(\begin{array}{ccc}
m & 0 & s \\
n & 0 & l
\end{array}\right)=E\left(\begin{array}{ccc}
m & s & 0 \\
n & l & 0
\end{array}\right)=4 \pi\left[\frac{1}{(2 n+1)(2 l+1)}\right]^{1 / 2} \delta_{m,-s} \delta_{n l},
$$

leading to

$$
(s \mid r)_{\alpha l 0}^{\beta s 0}=\sqrt{(4 \pi)}(-1)^{l} \delta_{\beta,-s} \delta_{\alpha l}, \quad(s \mid r)_{\alpha 0 n}^{\beta 0 m}=\sqrt{(4 \pi)} \delta_{\beta m} \delta_{\alpha n}, \quad(s \mid r)_{0 l n}^{0 s m}=\sqrt{(4 \pi)} \delta_{s m} \delta_{l n},
$$


Substituting (4.8) in (4.4) we have the following expression

$$
S_{n}^{m}\left(\mathbf{r}_{q}+\mathbf{r}_{p q}^{\prime}\right)=\sum_{l=0}^{\infty} \sum_{s=-l}^{l} \sum_{\alpha=0}^{\infty} \sum_{\beta=-\alpha}^{\alpha}(s \mid r)_{\alpha l n}^{\beta s m} S_{\alpha}^{\beta}\left(\mathbf{r}_{p q}^{\prime}\right) R_{l}^{s}\left(\mathbf{r}_{q}\right)
$$

which is a form of the addition theorem for multipole solutions of the Helmholtz equation. Similar considerations for the other reexpansion pairs yields:

$$
(R \mid R)_{l n}^{s m}\left(\mathbf{r}_{p q}^{\prime}\right)=\sum_{\alpha=0}^{\infty} \sum_{\beta=-\alpha}^{\alpha}(r \mid r)_{\alpha l n}^{\beta s m} R_{\alpha}^{\beta}\left(\mathbf{r}_{p q}^{\prime}\right), \quad(S \mid S)_{l n}^{s m}\left(\mathbf{r}_{p q}^{\prime}\right)=\sum_{\alpha=0}^{\infty} \sum_{\beta=-\alpha}^{\alpha}(s \mid s)_{\alpha l n}^{\beta s m} R_{\alpha}^{\beta}\left(\mathbf{r}_{p q}^{\prime}\right),
$$

This leads to the following addition theorems

$$
\begin{aligned}
& R_{n}^{m}\left(\mathbf{r}_{q}+\mathbf{r}_{p q}^{\prime}\right)=\sum_{l=0}^{\infty} \sum_{s=-l}^{l} \sum_{\alpha=0}^{\infty} \sum_{\beta=-\alpha}^{\alpha}(r \mid r)_{\alpha l n}^{\beta s m} R_{\alpha}^{\beta}\left(\mathbf{r}_{p q}^{\prime}\right) R_{l}^{s}\left(\mathbf{r}_{q}\right), \\
& S_{n}^{m}\left(\mathbf{r}_{q}+\mathbf{r}_{p q}^{\prime}\right)=\sum_{l=0}^{\infty} \sum_{s=-l}^{l} \sum_{\alpha=0}^{\infty} \sum_{\beta=-\alpha}^{\alpha}(s \mid s)_{\alpha l n}^{\beta s m} R_{\alpha}^{\beta}\left(\mathbf{r}_{p q}^{\prime}\right) S_{l}^{s}\left(\mathbf{r}_{q}\right),
\end{aligned}
$$

Comparing (4.20) with (4.17), we see that these are indeed the same expansions of $S_{n}^{m}\left(\mathbf{r}_{q}+\mathbf{r}_{p q}^{\prime}\right)$. We can simply transform one to the other by exchanging $\mathbf{r}_{p q}^{\prime}$ with $\mathbf{r}_{q}$ and subscripts $\alpha$ with $l$ and $\beta$ with $s$. Therefore,

$$
(s \mid s)_{\alpha \ln }^{\beta s m}=(s \mid r)_{l \alpha n}^{s \beta m}
$$

The numerical coef cients $(r \mid r)_{\alpha l n}^{\beta s m}$ can be also related to the Wigner symbols in a manner similar to the expression for $(s \mid r)_{\alpha \ln }^{\beta s m}$ (4.13). As will follow from our analysis below,

$$
(S \mid S)_{l n}^{s m}\left(\mathbf{r}_{p q}^{\prime}\right)=(R \mid R)_{l n}^{s m}\left(\mathbf{r}_{p q}^{\prime}\right)
$$

and thus from (4.18), and (4.21) we have:

$$
(r \mid r)_{\alpha l n}^{\beta s m}=(s \mid s)_{\alpha l n}^{\beta s m}=(s \mid r)_{l \alpha n}^{s \beta m} .
$$

However, even if the Wigner coef cients could be computed ef ciently, calculation of the reexpansion coef cients $(S \mid R)_{l n}^{s m},(R \mid R)_{l n}^{s m}$, and $(S \mid S)_{l n}^{s m}$ using them would require summation of series (4.8) and (4.18), which would be computationally expensive, since the reexpansion coef cients are 4-dimensional (and numerical coef cients, such as $(s \mid r)_{\alpha l n}^{\beta s m}$ are 5-dimensional (taking in account the relation (4.14)), leading to $O\left(N_{t}^{5}\right)$ operations. As an alternative method we develop a fast computational technique based on recurrent computation of the actual reexpansion coef cients.

4.3. Recurrence Relations for Translation Coef cients. Recurrence relations among the fundamental solutions of the Helmholtz equation produce recurrence relations for the reexpansion coef cients due to invariance of the differential operators $\partial / \partial z, \partial / \partial x \pm i \partial / \partial y$ with respect to translations of the origin of the reference frame. Since $S_{n}^{m}$ and $R_{n}^{m}$ satisfy the same recurrence relations, the reexpansion coef cients $(S \mid R)_{l n}^{s m},(S \mid S)_{l n}^{s m}$ and $(R \mid R)_{l n}^{s m}$ also satisfy the same recurrence relations. To avoid repeating theorems and recurrence relations for every combination of regular and singular functions, we denote the generic translation coef cient as $(E \mid F)_{l n}^{s m}\left(\mathbf{r}_{p q}^{\prime}\right)$ for any of the reexpansion coef cients $((E \mid F)=(S \mid R),(S \mid S)$ or $(R \mid R))$, i.e., $E$ and $F$ can be any of the functions $S$ or $R$. Thus the following reexpansion holds:

$$
E_{n}^{m}\left(\mathbf{r}_{p}\right)=E_{n}^{m}\left(\mathbf{r}_{q}+\mathbf{r}_{p q}^{\prime}\right)=\sum_{l=0}^{\infty} \sum_{s=-l}^{l}(E \mid F)_{l n}^{s m}\left(\mathbf{r}_{p q}^{\prime}\right) F_{l}^{s}\left(\mathbf{r}_{q}\right), \quad E, F=S, R .
$$

Denoting by $D_{p}$ any of operators $\partial / \partial z_{p}, \partial / \partial x_{p} \pm i \partial / \partial y_{p}$ in the reference frame with the origin at $\mathbf{r}_{p}^{\prime}$ and applying the operator to (4.24) at xed $\mathbf{r}_{p q}^{\prime}$, we have:

$$
D_{p} E_{n}^{m}\left(\mathbf{r}_{p}\right)=D_{q} E_{n}^{m}\left(\mathbf{r}_{q}+\mathbf{r}_{p q}^{\prime}\right)=\sum_{l=0}^{\infty} \sum_{s=-l}^{l}(E \mid F)_{l n}^{s m}\left(\mathbf{r}_{p q}^{\prime}\right) D_{q} F_{l}^{s}\left(\mathbf{r}_{q}\right), \quad E, F=S, R .
$$


The following theorems establish general recurrence relations. Their proofs are based on the theorems for differentiation of multipoles. We provide the proof only for the rst theorem. The other theorems can be proved in a similar way, or may be obtained from [2]. These relations, presumably rst, were obtained by Chew[22].

THEOREM 4.1. For $k \neq 0$ the following recurrence relation holds for $(E \mid F)_{l n}^{s m}\left(\mathbf{r}_{p q}^{\prime}\right)$ :

$$
\begin{gathered}
a_{n-1}^{m}(E \mid F)_{l, n-1}^{s m}\left(\mathbf{r}_{p q}^{\prime}\right)-a_{n}^{m}(E \mid F)_{l, n+1}^{s m}\left(\mathbf{r}_{p q}^{\prime}\right)=a_{l}^{s}(E \mid F)_{l+1, n}^{s m}\left(\mathbf{r}_{p q}^{\prime}\right)-a_{l-1}^{s}(E \mid F)_{l-1, n}^{s m}\left(\mathbf{r}_{p q}^{\prime}\right), \\
E, F=S, R, \quad l, n=0,1, \ldots \quad s=-l, \ldots, l, \quad m=-n, \ldots, n .
\end{gathered}
$$

Proof. Consider $D_{p}=k^{-1} \partial / \partial z_{p}=k^{-1} \partial / \partial z_{q}=D_{q}$. Using Theorem 3.1, (3.2), and (4.24) we nd

$$
\begin{aligned}
\frac{1}{k} \frac{\partial}{\partial z_{p}} E_{n}^{m}\left(\mathbf{r}_{p}\right) & =a_{n-1}^{m} E_{n-1}^{m}\left(\mathbf{r}_{p}\right)-a_{n}^{m} E_{n+1}^{m}\left(\mathbf{r}_{p}\right) \\
& =\sum_{l=0}^{\infty} \sum_{s=-l}^{l}\left[a_{n-1}^{m}(E \mid F)_{l, n-1}^{s m}\left(\mathbf{r}_{p q}^{\prime}\right)-a_{n}^{m}(E \mid F)_{l, n+1}^{s m}\left(\mathbf{r}_{p q}^{\prime}\right)\right] F_{l}^{s}\left(\mathbf{r}_{q}\right) .
\end{aligned}
$$

On the other hand using the same Theorem 3.1 and de nition we have

$$
\begin{aligned}
\frac{1}{k} \frac{\partial}{\partial z_{p}} E_{n}^{m}\left(\mathbf{r}_{p}\right) & =\sum_{l=0}^{\infty} \sum_{s=-l}^{l}(E \mid F)_{l n}^{s m}\left(\mathbf{r}_{p q}^{\prime}\right) \frac{1}{k} \frac{\partial}{\partial z_{q}} F_{l}^{s}\left(\mathbf{r}_{q}\right)=\sum_{l=0}^{\infty} \sum_{s=-l}^{l}(E \mid F)_{l n}^{s m}\left(\mathbf{r}_{p q}^{\prime}\right)\left[a_{l-1}^{s} F_{l-1}^{s}\left(\mathbf{r}_{q}\right)-a_{l}^{s} F_{l+1}^{s}\left(\mathbf{r}_{q}\right)\right] \\
& =\sum_{l=-1}^{\infty} \sum_{s=-l-1}^{l+1} a_{l}^{s}(E \mid F)_{l+1, n}^{s m}\left(\mathbf{r}_{p q}^{\prime}\right) F_{l}^{s}\left(\mathbf{r}_{q}\right)-\sum_{l=1}^{\infty} \sum_{s=-l+1}^{l-1} a_{l-1}^{s}(E \mid F)_{l-1, n}^{s m}\left(\mathbf{r}_{p q}^{\prime}\right) F_{l}^{s}\left(\mathbf{r}_{q}\right) \\
& =\sum_{l=0}^{\infty} \sum_{s=-l}^{l}\left[a_{l}^{s}(E \mid F)_{l+1, n}^{s m}\left(\mathbf{r}_{p q}^{\prime}\right)-a_{l-1}^{s}(E \mid F)_{l-1, n}^{s m}\left(\mathbf{r}_{p q}^{\prime}\right)\right] F_{l}^{s}\left(\mathbf{r}_{q}\right) .
\end{aligned}
$$

The last equality holds due to according de nition (3.3)

$$
a_{-1}^{s}=a_{l}^{l+1}=a_{l}^{-l-1}=a_{l-1}^{l}=a_{l-1}^{-l}=0 .
$$

Comparing these two expressions and using the orthogonality and completeness of the surface harmonics we obtain the statement of the theorem.

COROLlary 4.2. For $n=|m|$ :

$$
\begin{aligned}
a_{|m|}^{m}(E \mid F)_{l,|m|+1}^{s m}\left(\mathbf{r}_{p q}^{\prime}\right) & =a_{l-1}^{s}(E \mid F)_{l-1,|m|}^{s m}\left(\mathbf{r}_{p q}^{\prime}\right)-a_{l}^{s}(E \mid F)_{l+1,|m|}^{s m}\left(\mathbf{r}_{p q}^{\prime}\right), \quad E, F=S, R, \\
l & =0,1, \ldots \quad s=-l, \ldots, l, \quad m=0, \pm 1, \pm 2, \ldots
\end{aligned}
$$

For $l=|s|$ :

$$
\begin{aligned}
a_{|s|}^{s}(E \mid F)_{|s|+1, n}^{s m}\left(\mathbf{r}_{p q}^{\prime}\right) & =a_{n-1}^{m}(E \mid F)_{|s|, n-1}^{s m}\left(\mathbf{r}_{p q}^{\prime}\right)-a_{n}^{m}(E \mid F)_{|s|, n+1}^{s m}\left(\mathbf{r}_{p q}^{\prime}\right), \quad E, F=S, R, \\
n & =0,1, \ldots \quad m=-n, \ldots, n \quad s=0, \pm 1, \pm 2, \ldots
\end{aligned}
$$

For $n=|m|$ and $l=|s|$ :

$$
\begin{aligned}
a_{|s|}^{s}(E \mid F)_{|s|+1,|m|}^{s m}\left(\mathbf{r}_{p q}^{\prime}\right) & =-a_{|m|}^{m}(E \mid F)_{|s|,|m|+1}^{s m}\left(\mathbf{r}_{p q}^{\prime}\right), \quad E, F=S, R, \\
m & =0, \pm 1, \pm 2, \ldots \quad s=0, \pm 1, \pm 2, \ldots
\end{aligned}
$$

THEOREM 4.3. For $k \neq 0$ the following recurrence relation holds for $(E \mid F)_{l n}^{s m}\left(\mathbf{r}_{p q}^{\prime}\right)$ :

$$
\begin{gathered}
b_{n}^{m}(E \mid F)_{l, n-1}^{s, m+1}\left(\mathbf{r}_{p q}^{\prime}\right)-b_{n+1}^{-m-1}(E \mid F)_{l, n+1}^{s, m+1}\left(\mathbf{r}_{p q}^{\prime}\right)=b_{l+1}^{s-1}(E \mid F)_{l+1, n}^{s-1, m}\left(\mathbf{r}_{p q}^{\prime}\right)-b_{l}^{-s}(E \mid F)_{l-1, n}^{s-1, m}\left(\mathbf{r}_{p q}^{\prime}\right), \\
E, F=S, R, \quad l, n=0,1, \ldots \quad s=-l, \ldots, l, \quad m=-n, \ldots, n .
\end{gathered}
$$


Corollary 4.4. For $n=m$

$$
\begin{aligned}
b_{m+1}^{-m-1}(E \mid F)_{l, m+1}^{s, m+1}\left(\mathbf{r}_{p q}^{\prime}\right) & =b_{l}^{-s}(E \mid F)_{l-1, m}^{s-1, m}\left(\mathbf{r}_{p q}^{\prime}\right)-b_{l+1}^{s-1}(E \mid F)_{l+1, m}^{s-1, m}\left(\mathbf{r}_{p q}^{\prime}\right) \\
E, F & =S, R, \quad l=0,1, \ldots \quad s=-l, \ldots, l, \quad m=0,1,2, \ldots
\end{aligned}
$$

For $l=|s|, s \leqslant 0$

$$
\begin{aligned}
b_{|s|+1}^{-|s|-1}(E \mid F)_{|s|+1, n}^{-|s|-1, m}\left(\mathbf{r}_{p q}^{\prime}\right) & =b_{n}^{m}(E \mid F)_{|s|, n-1}^{-|s|, m+1}\left(\mathbf{r}_{p q}^{\prime}\right)-b_{n+1}^{-m-1}(E \mid F)_{|s|, n+1}^{-|s|, m+1}\left(\mathbf{r}_{p q}^{\prime}\right) \\
E, F & =S, R, \quad n=0,1, \ldots \quad m=-n, \ldots, n \quad s=0,-1,-2, \ldots
\end{aligned}
$$

For $n=m$ and $l=|s|, s \leqslant 0$

$$
\begin{aligned}
b_{m+1}^{-m-1}(E \mid F)_{|s|, m+1}^{-|s|, m+1}\left(\mathbf{r}_{p q}^{\prime}\right) & =-b_{|s|+1}^{-|s|-1}(E \mid F)_{|s|+1, m}^{-|s|-1, m}\left(\mathbf{r}_{p q}^{\prime}\right), \\
E, F & =S, R, \quad m=0,1,2, \ldots \quad s=0,-1,-2, \ldots
\end{aligned}
$$

THEOREM 4.5. For $k \neq 0$ the following recurrence relation holds for $(E \mid F)_{l n}^{s m}\left(\mathbf{r}_{p q}^{\prime}\right)$ :

$$
\begin{gathered}
b_{n+1}^{m-1}(E \mid F)_{l, n+1}^{s, m-1}\left(\mathbf{r}_{p q}^{\prime}\right)-b_{n}^{-m}(E \mid F)_{l, n-1}^{s, m-1}\left(\mathbf{r}_{p q}^{\prime}\right)=b_{l}^{s}(E \mid F)_{l-1, n}^{s+1, m}\left(\mathbf{r}_{p q}^{\prime}\right)-b_{l+1}^{-s-1}(E \mid F)_{l+1, n}^{s+1, m}\left(\mathbf{r}_{p q}^{\prime}\right), \\
E, F=S, R, \quad l, n=0,1, \ldots \quad s=-l, \ldots, l, \quad m=-n, \ldots, n
\end{gathered}
$$

COROLlary 4.6. For $n=|m|, m \leqslant 0$

$$
\begin{aligned}
b_{|m|+1}^{-|m|-1}(E \mid F)_{l,|m|+1}^{s,-|m|-1}\left(\mathbf{r}_{p q}^{\prime}\right) & =b_{l}^{s}(E \mid F)_{l-1,|m|}^{s+1,-|m|}\left(\mathbf{r}_{p q}^{\prime}\right)-b_{l+1}^{-s-1}(E \mid F)_{l+1,|m|}^{s+1,-|m|}\left(\mathbf{r}_{p q}^{\prime}\right) \\
E, F & =S, R, \quad l=0,1, \ldots \quad s=-l, \ldots, l, \quad m=0,-1,-2, \ldots
\end{aligned}
$$

For $l=s$

$$
\begin{aligned}
b_{s+1}^{-s-1}(E \mid F)_{s+1, n}^{s+1, m}\left(\mathbf{r}_{p q}^{\prime}\right) & =b_{n}^{-m}(E \mid F)_{s, n-1}^{s, m-1}\left(\mathbf{r}_{p q}^{\prime}\right)-b_{n+1}^{m-1}(E \mid F)_{s, n+1}^{s, m-1}\left(\mathbf{r}_{p q}^{\prime}\right) \\
E, F & =S, R, \quad n=0,1, \ldots \quad m=-n, \ldots, n \quad s=0,1,2, \ldots
\end{aligned}
$$

For $n=|m|, m \leqslant 0$ and $l=s$

$$
\begin{aligned}
b_{|m|+1}^{-|m|-1}(E \mid F)_{s,|m|+1}^{s,-|m|-1}\left(\mathbf{r}_{p q}^{\prime}\right) & =-b_{s+1}^{-s-1}(E \mid F)_{s+1,|m|}^{s+1,-|m|}\left(\mathbf{r}_{p q}^{\prime}\right), \quad E, F=S, R, \\
m & =0,-1,-2, \ldots \quad s=0,1,2, \ldots
\end{aligned}
$$

4.4. Particular Values of Translation Coef cients. To use the recurrence relations we need some starting values. The following particular values provide these.

4.4.1. ( $\mathbf{S} \mid \mathbf{R})$ Coef cients. Expression (4.17) reveals a particular value of the reexpansion coef cients $(S \mid R)_{l n}^{s m}$. Setting $\mathbf{r}_{q}=\mathbf{0}$ we have

$$
R_{l}^{s}(\mathbf{0})=\sqrt{\frac{1}{4 \pi}} \delta_{l 0} \delta_{s 0}, \quad S_{n}^{m}\left(\mathbf{r}_{p q}^{\prime}\right)=\sqrt{\frac{1}{4 \pi}} \sum_{\alpha=0}^{\infty} \sum_{\beta=-\alpha}^{\alpha}(s \mid r)_{\alpha 0 n}^{\beta 0 m} S_{\alpha}^{\beta}\left(\mathbf{r}_{p q}^{\prime}\right), \quad p \neq q .
$$

Due to the orthogonality of the surface harmonics

$$
(s \mid r)_{\alpha 0 n}^{\beta 0 m}=\sqrt{(4 \pi)} \delta_{\alpha n} \delta_{\beta m}
$$


This value also can be obtained directly from (4.16). Substituting this expression in (4.8), we have

$$
(S \mid R)_{0 n}^{0 m}\left(\mathbf{r}_{p q}^{\prime}\right)=\sqrt{(4 \pi)} S_{n}^{m}\left(\mathbf{r}_{p q}^{\prime}\right), \quad n=0,1, \ldots, \quad m=-n, \ldots, n .
$$

Another particular value can be found from well-known expansion of fundamental solution $G\left(\mathbf{r}_{p}\right)$ of the Helmholtz equation [5] in a series of spherical harmonics:

$$
G\left(\mathbf{r}_{p}\right)=i k \sum_{l=0}^{\infty} \sum_{s=-l}^{s=l} S_{l}^{-s}\left(-\mathbf{r}_{p q}^{\prime}\right) R_{l}^{s}\left(\mathbf{r}_{q}\right), \quad\left|\mathbf{r}_{q}\right| \leqslant\left|\mathbf{r}_{p q}^{\prime}\right| .
$$

We recall the fundamental solution is a monopole

$$
G\left(\mathbf{r}_{p}\right)=\frac{e^{i k r_{p}}}{4 \pi r_{p}}=\frac{i k}{4 \pi} h_{0}\left(k r_{p}\right)=\frac{i k}{\sqrt{(4 \pi)}} S_{0}^{0}\left(\mathbf{r}_{q}+\mathbf{r}_{p q}^{\prime}\right)
$$

Thus, comparing (4.41) and (4.42) with (4.4), we obtain the following value for the reexpansion coef cients:

$$
(S \mid R)_{l 0}^{s 0}\left(\mathbf{r}_{p q}^{\prime}\right)=\sqrt{(4 \pi)} S_{l}^{-s}\left(-\mathbf{r}_{p q}^{\prime}\right)=\sqrt{(4 \pi)}(-1)^{l} S_{l}^{-s}\left(\mathbf{r}_{p q}^{\prime}\right), \quad l=0,1, \ldots, \quad s=-l, \ldots, l .
$$

Comparing (4.43) with (4.8), we have

$$
(s \mid r)_{\alpha l 0}^{\beta s 0}=\sqrt{(4 \pi)}(-1)^{l} \delta_{\alpha l} \delta_{\beta,-s},
$$

which is consistent with (4.16).

Note, that formulae (4.40) and (4.43) are consistent as they both provide the value of $(S \mid R)_{00}^{00}\left(\mathbf{r}_{p q}^{\prime}\right)$ as

$$
(S \mid R)_{00}^{00}\left(\mathbf{r}_{p q}^{\prime}\right)=\sqrt{(4 \pi)} S_{0}^{0}\left(\mathbf{r}_{p q}^{\prime}\right)=h_{0}\left(k r_{p q}^{\prime}\right) .
$$

It is also worth mentioning that once $(S \mid R)_{l n}^{s m}\left(\mathbf{r}_{p q}^{\prime}\right)$ is known, the coef cients $(S \mid R)_{l n}^{s m}\left(\mathbf{r}_{q p}^{\prime}\right)$ representing reexpansion of multipoles near point $p$,

$$
S_{n}^{m}\left(\mathbf{r}_{q}\right)=\sum_{l=0}^{\infty} \sum_{s=-l}^{l}(S \mid R)_{l n}^{s m}\left(\mathbf{r}_{q p}^{\prime}\right) R_{l}^{s}\left(\mathbf{r}_{p}\right), \quad p \neq q,
$$

can be determined due to a symmetry relation. Indeed, changing the sign of the radius vector in (4.4) we have:

$$
\begin{array}{r}
\sum_{l=0}^{\infty} \sum_{s=-l}^{l}(S \mid R)_{l n}^{s m}\left(\mathbf{r}_{p q}^{\prime}\right) R_{l}^{s}\left(\mathbf{r}_{q}\right)=S_{n}^{m}\left(\mathbf{r}_{q}+\mathbf{r}_{p q}^{\prime}\right)=(-1)^{n} S_{n}^{m}\left(-\mathbf{r}_{q}-\mathbf{r}_{p q}^{\prime}\right) \\
=(-1)^{n} \sum_{l=0}^{\infty} \sum_{s=-l}^{l}(S \mid R)_{l n}^{s m}\left(-\mathbf{r}_{p q}^{\prime}\right) R_{l}^{s}\left(-\mathbf{r}_{q}\right)=\sum_{l=0}^{\infty} \sum_{s=-l}^{l}(-1)^{n+l}(S \mid R)_{l n}^{s m}\left(\mathbf{r}_{q p}^{\prime}\right) R_{l}^{s}\left(\mathbf{r}_{q}\right) .
\end{array}
$$

Due to orthogonality of surface harmonics we obtain:

$$
\begin{aligned}
(S \mid R)_{l n}^{s m}\left(\mathbf{r}_{q p}^{\prime}\right) & =(-1)^{n+l}(S \mid R)_{l n}^{s m}\left(\mathbf{r}_{p q}^{\prime}\right), \quad p \neq q, \\
n, l & =0,1, \ldots, \quad m=-n, \ldots, n, \quad l=-s, \ldots, s .
\end{aligned}
$$

We also can nd using (4.4) and

$$
S_{n}^{m}=0, \quad R_{n}^{m}=0, \quad \text { for }|m|>n,
$$

that

$$
(S \mid R)_{l n}^{s m}\left(\mathbf{r}_{p q}^{\prime}\right)=0, \quad \text { for }|m|>n \text { or }|s|>l .
$$


4.4.2. (R|R) Coef cients. Setting $\mathbf{r}_{q}=\mathbf{0}$ in (4.19) and using (4.38) we have

$$
R_{n}^{m}\left(\mathbf{r}_{p q}^{\prime}\right)=\sqrt{\frac{1}{4 \pi}} \sum_{\alpha=0}^{\infty} \sum_{\beta=-\alpha}^{\alpha}(r \mid r)_{\alpha 0 n}^{\beta 0 m} R_{\alpha}^{\beta}\left(\mathbf{r}_{p q}^{\prime}\right) .
$$

Due to the orthogonality of the surface harmonics we obtain

$$
(r \mid r)_{\alpha 0 n}^{\beta 0 m}=\sqrt{(4 \pi)} \delta_{\alpha n} \delta_{\beta m}
$$

Substituting this expression in (4.49), we have

$$
(R \mid R)_{0 n}^{0 m}\left(\mathbf{r}_{p q}^{\prime}\right)=\sqrt{(4 \pi)} R_{n}^{m}\left(\mathbf{r}_{p q}^{\prime}\right), \quad n=0,1, \ldots, \quad m=-n, \ldots, n .
$$

In (4.19) we also can set $\mathbf{r}_{p q}^{\prime}=\mathbf{0}$. In this case from (4.38) we have

$$
R_{n}^{m}\left(\mathbf{r}_{q}\right)=\sqrt{\frac{1}{4 \pi}} \sum_{l=0}^{\infty} \sum_{s=-l}^{l}(r \mid r)_{0 l n}^{0 s m} R_{l}^{s}\left(\mathbf{r}_{q}\right) .
$$

This yields

$$
(r \mid r)_{0 l n}^{0 s m}=\sqrt{(4 \pi)} \delta_{l n} \delta_{s m} .
$$

Both values (4.50) and (4.53) are consistent with those following from the particular values of the Wigner symbols (4.16). It may also be noted that (4.19) is symmetrical with respect to the exchange of $\mathbf{r}_{q}$ and $\mathbf{r}_{p q}^{\prime}$. This leads to the following symmetry relation

$$
(r \mid r)_{\alpha l n}^{\beta s m}=(r \mid r)_{l \alpha n}^{s \beta m} .
$$

To obtain the value of $(R \mid R)_{l 0}^{s 0}\left(\mathbf{r}_{p q}^{\prime}\right)$ we note that the spherical Bessel and Hankel functions of the 1st kind are related by

$$
j_{n}(k r)=\frac{1}{2}\left[h_{n}(k r)+\overline{h_{n}(k r)}\right] .
$$

Particularly for $n=0$ this results in

$$
R_{0}^{0}\left(\mathbf{r}_{q}\right)=\frac{1}{2}\left[S_{0}^{0}\left(\mathbf{r}_{q}\right)+\overline{S_{0}^{0}\left(\mathbf{r}_{q}\right)}\right] .
$$

Using this relation and the expansion of the fundamental solution (4.41), (4.42), and (4.55) we obtain:

$$
R_{0}^{0}\left(\mathbf{r}_{q}\right)=\sqrt{(4 \pi)} \sum_{l=0}^{\infty} \sum_{s=-l}^{l}(-1)^{l} R_{l}^{-s}\left(\mathbf{r}_{p q}^{\prime}\right) R_{l}^{s}\left(\mathbf{r}_{q}\right) .
$$

Comparing this expansion with (2.21) and using the orthogonality of the surface harmonics, we obtain

$$
(R \mid R)_{l 0}^{s 0}\left(\mathbf{r}_{p q}^{\prime}\right)=\sqrt{(4 \pi)}(-1)^{l} R_{l}^{-s}\left(\mathbf{r}_{p q}^{\prime}\right), \quad l=0,1, \ldots, \quad s=-l, \ldots, l .
$$

In the same way as for $(S \mid R)_{l n}^{s m}\left(\mathbf{r}_{p q}^{\prime}\right)$ (see (4.46) and (4.48)) we can show that

$$
\begin{aligned}
& (R \mid R)_{l n}^{s m}\left(\mathbf{r}_{q p}^{\prime}\right)=(-1)^{n+l}(R \mid R)_{l n}^{s m}\left(\mathbf{r}_{p q}^{\prime}\right), \quad n, l=0,1, \ldots, \quad m=-n, \ldots, n, \quad l=-s, \ldots, s \\
& (R \mid R)_{l n}^{s m}\left(\mathbf{r}_{p q}^{\prime}\right)=0, \quad \text { for }|m|>n \text { or }|s|>l .
\end{aligned}
$$

Note that to obtain the above values and properties of coef cients $(S \mid R)_{l n}^{s m}$ and $(R \mid R)_{l n}^{s m}$ there is no need to use the Wigner or Clebsch-Gordan coef cients. 
4.5. Symmetry of Translation Coef cients. The reexpansion coef cients obey many symmetry properties, which can be a subject for a separate publication and study. These symmetry relations are very important for ef cient computation as they enable evaluation of all coef cients by computing only a few of then, and are important for developing fast numerical methods. Here we just mention the following symmetries (the proofs can be found in [2]):

THEOREM 4.7. The following symmetry relation holds:

$$
(E \mid F)_{l n}^{s m}\left(\mathbf{r}_{p q}^{\prime}\right)=(-1)^{n+l}(E \mid F)_{n l}^{-m,-s}\left(\mathbf{r}_{p q}^{\prime}\right), \quad E, F=S, R, \quad l, n=0,1, \ldots \quad s=-l, \ldots, l, \quad m=-n, \ldots, n .
$$

THEOREM 4.8. The following symmetry relation holds:

$$
(R \mid R)_{l n}^{s m}\left(\mathbf{r}_{p q}^{\prime}\right)=\overline{(R \mid R)_{l n}^{-s,-m}\left(\mathbf{r}_{p q}^{\prime}\right)}, \quad l, n=0,1, \ldots \quad s=-l, \ldots, l, \quad m=-n, \ldots, n .
$$

THEOREM 4.9. The translation reexpansion coef cients $(R \mid R)_{l n}^{s m}\left(\mathbf{r}_{p q}^{\prime}\right)$ and $(S \mid S)_{l n}^{s m}\left(\mathbf{r}_{p q}^{\prime}\right)$ are the same:

$$
(S \mid S)_{l n}^{s m}\left(\mathbf{r}_{p q}^{\prime}\right)=(R \mid R)_{l n}^{s m}\left(\mathbf{r}_{p q}^{\prime}\right), \quad l, n=0,1, \ldots \quad s=-l, \ldots, l, \quad m=-n, \ldots, n .
$$

4.6. Sectorial Translation Coef cients. In analogy with the surface spherical harmonics we will call reexpansion coef cients of the type $(E \mid F)_{l|m|}^{s m}$ and $(E \mid F)_{|s| n}^{s m}$ as "sectorial reexpansion" coef cients, since they involve reexpansion of sectorial harmonics or represent coef cients near sectorial harmonics in reexpansions. For such coef cients we will use simpli ed notation

$$
(E \mid F)_{l,}^{s m}=(E \mid F)_{l|m|}^{s m}, \quad(E \mid F)_{, n}^{s m}=(E \mid F)_{|s| n}^{s m}, \quad E, F=S, R
$$

Particularly we have from (4.40), (4.43) and (4.51), (4.58):

$$
\begin{aligned}
(S \mid R)_{l,}^{s 0}\left(\mathbf{r}_{p q}^{\prime}\right)=\sqrt{(4 \pi)}(-1)^{l} S_{l}^{-s}\left(\mathbf{r}_{p q}^{\prime}\right), & & (S \mid R)_{, n}^{0 m}\left(\mathbf{r}_{p q}^{\prime}\right)=\sqrt{(4 \pi)} S_{n}^{m}\left(\mathbf{r}_{p q}^{\prime}\right), \\
(R \mid R)_{l,}^{s 0}\left(\mathbf{r}_{p q}^{\prime}\right)=\sqrt{(4 \pi)}(-1)^{l} R_{l}^{-s}\left(\mathbf{r}_{p q}^{\prime}\right), & & (R \mid R)_{, n}^{0 m}\left(\mathbf{r}_{p q}^{\prime}\right)=\sqrt{(4 \pi)} R_{n}^{m}\left(\mathbf{r}_{p q}^{\prime}\right) .
\end{aligned}
$$

We will call the coef cients $(E \mid F)_{|s||m|}^{s m}$ as "double sectorial reexpansion" coef cients and simplify notation as

$$
(E \mid F)^{s m}=(E \mid F)_{|s||m|}^{s m}, \quad E, F=S, R .
$$

Particularly, (4.64) provides:

$$
\begin{aligned}
& (S \mid R)^{s 0}\left(\mathbf{r}_{p q}^{\prime}\right)=\sqrt{(4 \pi)}(-1)^{s} S_{l}^{-s}\left(\mathbf{r}_{p q}^{\prime}\right), \quad(S \mid R)^{0 m}\left(\mathbf{r}_{p q}^{\prime}\right)=\sqrt{(4 \pi)} S_{n}^{m}\left(\mathbf{r}_{p q}^{\prime}\right), \\
& (R \mid R)^{s 0}\left(\mathbf{r}_{p q}^{\prime}\right)=\sqrt{(4 \pi)}(-1)^{s} R_{l}^{-s}\left(\mathbf{r}_{p q}^{\prime}\right), \quad(R \mid R)^{0 m}\left(\mathbf{r}_{p q}^{\prime}\right)=\sqrt{(4 \pi)} R_{n}^{m}\left(\mathbf{r}_{p q}^{\prime}\right) .
\end{aligned}
$$

The reason why we pay special attention to the sectorial reexpansion coef cients is that they are either known explicitly or can be computed via simple recurrence relations, and thus can be used as "boundary conditions" (i.e., initial values) for the recursive computation of the tesseral reexpansion coef cients $(E \mid F)_{l n}^{s m}, E, F=S, R$.

4.6.1. Computation of Sectorial Translation Coef cients. The sectorial reexpansion coef cients can be computed independently from the other coef cients, since the initial values (4.64) and recurrence relations (4.31)-(4.32) and (4.35)-(4.36) include only sectorial coef cients and are suf cient for their computation. Note that only coef cients $(E \mid F)_{l,}^{s m}$ can be computed while $(E \mid F)_{, l}^{s m}$ can be determined using symmetry (4.61). These relations can be rewritten in the form:

$$
\begin{gathered}
b_{m+1}^{-m-1}(E \mid F)_{l,}^{s, m+1}=b_{l}^{-s}(E \mid F)_{l-1,}^{s-1, m}-b_{l+1}^{s-1}(E \mid F)_{l+1,}^{s-1, m}, l=0,1, \ldots \quad s=-l, \ldots, l, \quad m=0,1,2, \ldots \\
b_{m+1}^{-m-1}(E \mid F)_{l,}^{s,-m-1}=b_{l}^{s}(E \mid F)_{l-1,}^{s+1,-m}-b_{l+1}^{-s-1}(E \mid F)_{l+1,}^{s+1,-m}, \quad E, F=S, R \\
\quad l=0,1, \ldots \quad s=-l, \ldots, l, \quad m=0,1,2, \ldots
\end{gathered}
$$


Relations (4.67) and (4.68) provide values of coef cients $(E \mid F)_{l,}^{s m}$ for layers with increasing $|m|$. This process starts with known values $(E \mid F)_{l,}^{s 0}$ (4.64).

Note also the following relation for the sectorial reexpansion coef cients following from (4.29)

$$
\begin{aligned}
a_{|s|}^{s}(E \mid F)_{|s|+1}^{s m} & =-a_{|m|}^{m}(E \mid F)_{,|m|+1}^{s m}, \quad E, F=S, R, \\
m & =0, \pm 1, \pm 2, \ldots \quad s=0, \pm 1, \pm 2, \ldots
\end{aligned}
$$

4.6.2. Particular Values of Double Sectorial Translation Coef cients. Using the notation of (4.65) we can rewrite relation (4.33) in the form

$$
b_{m+1}^{-m-1}(E \mid F)^{-s, m+1}=-b_{s+1}^{-s-1}(E \mid F)^{-s-1, m}, \quad E, F=S, R, \quad m, s=0,1,2, \ldots
$$

Recursive application of this formula and values of coef cients $b_{m}^{-m}$ (3.5) enables expression of $(E \mid F)^{-s, m}$ coef cients $(m, s=0,1,2, \ldots)$ via $(E \mid F)^{-s-m, 0}$ :

$$
\begin{aligned}
(S \mid R)^{-s, m}\left(\mathbf{r}_{p q}^{\prime}\right) & =(-1)^{s} \frac{(s+m) !}{s ! m !} \sqrt{\frac{4 \pi(2 m+1) !(2 s+1) !}{(2 s+2 m+1) !}} S_{s+m}^{s+m}\left(\mathbf{r}_{p q}^{\prime}\right), \\
(R \mid R)^{-s, m}\left(\mathbf{r}_{p q}^{\prime}\right) & =(S \mid S)^{-s, m}\left(\mathbf{r}_{p q}^{\prime}\right)=(-1)^{s} \frac{(s+m) !}{s ! m !} \sqrt{\frac{4 \pi(2 m+1) !(2 s+1) !}{(2 s+2 m+1) !}} R_{s+m}^{s+m}\left(\mathbf{r}_{p q}^{\prime}\right), \\
m & =0,1,2, \ldots \quad s=0,1,2, \ldots
\end{aligned}
$$

Using this and symmetry relation (4.61) we can also determine $(S \mid R)^{s,-m}\left(\mathbf{r}_{p q}^{\prime}\right)$ and $(R \mid R)^{-s, m}\left(\mathbf{r}_{p q}^{\prime}\right)$.

4.7. Zonal Translation Coef cients. Using terminology, similar to that used for spherical surface harmonics (zonal, sectorial and tesseral harmonics) we call the coef cients

$$
(E \mid F)_{l n}\left(\mathbf{r}_{p q}^{\prime}\right)=(E \mid F)_{l n}^{00}\left(\mathbf{r}_{p q}^{\prime}\right), \quad E, F=S, R, \quad l, n=0,1,2, \ldots
$$

"zonal" reexpansion coef cients.

Particularly we have from (4.40) and (4.43), (4.51) and (4.58) :

$$
\begin{aligned}
& (S \mid R)_{l 0}\left(\mathbf{r}_{p q}^{\prime}\right)=\sqrt{(4 \pi)}(-1)^{l} S_{l}^{0}\left(\mathbf{r}_{p q}^{\prime}\right), \quad(S \mid R)_{0 n}\left(\mathbf{r}_{p q}^{\prime}\right)=\sqrt{(4 \pi)} S_{n}^{0}\left(\mathbf{r}_{p q}^{\prime}\right), \\
& (R \mid R)_{l 0}\left(\mathbf{r}_{p q}^{\prime}\right)=\sqrt{(4 \pi)}(-1)^{l} R_{l}\left(\mathbf{r}_{p q}^{\prime}\right), \quad(R \mid R)_{0 n}\left(\mathbf{r}_{p q}^{\prime}\right)=\sqrt{(4 \pi)} R_{n}^{m}\left(\mathbf{r}_{p q}^{\prime}\right) .
\end{aligned}
$$

As sectorial coef cients, the zonal reexpansion coef cients can be computed independently from the other coef cients using (4.26), which can be rewritten as

$$
a_{n-1}^{0}(E \mid F)_{l, n-1}-a_{n}^{0}(E \mid F)_{l, n+1}=a_{l}^{0}(E \mid F)_{l+1, n}-a_{l-1}^{0}(E \mid F)_{l-1, n}, \quad l, n=0,1, \ldots
$$

4.8. Coaxial Translation Coef cients. The translation coef cients derived above correspond to the case where the fundamental solutions are translated from one 3-D reference frame to another arbitrary 3-D reference frame. In some situations the second reference frame may not be arbitrary, and in these cases there can be a substantial simpli cation in the expressions and a signi cant reduction in the computation necessary for the reexpansion coef cients. We consider the case where the translation direction has its axis $z$ directed from point $\mathbf{r}_{p}^{\prime}$ to the center of reexpansion $\mathbf{r}_{q}^{\prime}$. Since, as discussed earlier, the reexpansion coef cients depend only on $\mathbf{r}_{p q}^{\prime}$, in this case the reexpansion coef cients will be independent of the angular variables. In these particular cases the general reexpansion formulae (2.19)-(2.21) simplify considerably to:

$$
\begin{array}{ll}
S_{n}^{m}\left(\mathbf{r}_{p}\right)=\sum_{l=|m|}^{\infty}(S \mid R)_{l n}^{m}\left(r_{p q}^{\prime}\right) R_{l}^{m}\left(\mathbf{r}_{q}\right), & \left|\mathbf{r}_{q}\right|<\left|\mathbf{r}_{p q}^{\prime}\right|, \\
S_{n}^{m}\left(\mathbf{r}_{p}\right)=\sum_{l=|m|}^{\infty}(S \mid S)_{l n}^{m}\left(r_{p q}^{\prime}\right) S_{l}^{m}\left(\mathbf{r}_{q}\right), & \left|\mathbf{r}_{q}\right|>\left|\mathbf{r}_{p q}^{\prime}\right| \\
R_{n}^{m}\left(\mathbf{r}_{p}\right)=\sum_{l=|m|}^{\infty}(R \mid R)_{l n}^{m}\left(r_{p q}^{\prime}\right) R_{l}^{m}\left(\mathbf{r}_{q}\right) . &
\end{array}
$$


Since the coef cients are now only three dimensional (three indices), we can say that they correspond to a "diagonalization" of the 4-D case. We call them "coaxial translation coef cients". These coef cients are related to the 4-index general translation coef cients.

$$
(E \mid F)_{l n}^{m}\left(r_{p q}^{\prime}\right)=\left.(E \mid F)_{l n}^{m m}\left(\mathbf{r}_{p q}^{\prime}\right)\right|_{\theta_{p q}^{\prime}=0}, \quad E, F=S, R, \quad l, n=0,1, \ldots, \quad m=-n, \ldots, n,
$$

also satisfy the general recurrence relations derived earlier and can could be computed using the algorithm for their computation. However, it is possible to derive simpler relations that allow for their fast computation.

The recurrence formula (4.26) does not act on the orders of the reexpansion coef cients, so setting $s=m$ there, we have

$a_{n-1}^{m}(E \mid F)_{l, n-1}^{m}-a_{n}^{m}(E \mid F)_{l, n+1}^{m}=a_{l}^{m}(E \mid F)_{l+1, n}^{m}-a_{l-1}^{m}(E \mid F)_{l-1, n}^{m}, \quad l, n=0,1, \ldots \quad m=-n, \ldots, n$.

In relation (4.30) we set $s=m+1$ to obtain

$$
b_{n}^{m}(E \mid F)_{l, n-1}^{m+1}-b_{n+1}^{-m-1}(E \mid F)_{l, n+1}^{m+1}=b_{l+1}^{m}(E \mid F)_{l+1, n}^{m}-b_{l}^{-m-1}(E \mid F)_{l-1, n}^{m}, \quad l, n=0,1, \ldots \quad m=-n, \ldots, n .
$$

Now it is obvious that we can start from $m=0$ and $(E \mid F)_{l n}^{0}$ to compute $(E \mid F)_{l n}^{m}$ and $(E \mid F)_{l n}^{-m}$ for $m=1,2, \ldots$ by using (4.80) and obtain all the coef cients. Since the recurrence coef cients in (4.80) for propagation in positive and negative directions of $m$ are the same, we come to the conclusion that

$$
(E \mid F)_{l n}^{m}=(E \mid F)_{l n}^{-m}=(E \mid F)_{l n}^{|m|}, \quad l, n=0,1, \ldots, \quad m=-n, \ldots, n .
$$

Therefore computation of $(E \mid F)_{l n}^{m}$ is required only for non-negative $m$ and formulae (4.79) and (4.80) are suf cient for this purpose. Due to (4.61) we also have the following symmetry property, enabling further ef ciencies

$$
(E \mid F)_{l n}^{m}=(-1)^{n+l}(E \mid F)_{n l}^{m}, \quad l, n=0,1, \ldots \quad m=-n, \ldots, n .
$$

4.8.1. Computation of the Coaxial Translation Coef cients. Due to the symmetry relations $(E \mid F)_{l n}^{m}$ can be computed only for $l \geqslant n \geqslant m \geqslant 0$. The process of recurrent computation of the coef cients $\left\{(E \mid F)_{l n}^{m}\right\}$ can be performed by computing the entries corresponding to the degrees $l$ and $n$ followed by advancement with respect to the order $m$. We need to initialize the procedure by providing values for $m=0$. According (4.78) and (4.64) we have

$$
\begin{aligned}
(S \mid R)_{l 0}^{0}\left(r_{p q}^{\prime}\right) & =\left.\sqrt{(4 \pi)}(-1)^{l} S_{l}^{0}\left(\mathbf{r}_{p q}^{\prime}\right)\right|_{\theta_{p q}^{\prime}=0}=(-1)^{l} \sqrt{(2 l+1)} h_{l}\left(k r_{p q}^{\prime}\right), \\
(R \mid R)_{l 0}^{0}\left(r_{p q}^{\prime}\right) & =(-1)^{l} \sqrt{(2 l+1)} j_{l}\left(k r_{p q}^{\prime}\right),
\end{aligned}
$$

For advancement with respect to $m$ it is convenient to use (4.80) for $n=m$ :

$$
b_{m+1}^{-m-1}(E \mid F)_{l, m+1}^{m+1}=b_{l}^{-m-1}(E \mid F)_{l-1, m}^{m}-b_{l+1}^{m}(E \mid F)_{l+1, m}^{m}, \quad l=m+1, m+2, \ldots
$$

and obtain other $(E \mid F)_{l n}^{m+1}$ using (4.79) and (4.82) in the same way as $(E \mid F)_{l n}^{0}$ are computed.

Formulae (4.84) and (4.83) employ sectorial coef cients of type $(E \mid F)_{l m}^{m}$, which can conveniently be denoted as

$$
(E \mid F)_{l}^{m}=(E \mid F)_{l m}^{m}, \quad l=m, m+1, \ldots
$$

which satisfy the relations

$$
\begin{aligned}
(S \mid R)_{l}^{0}= & (-1)^{l} \sqrt{(2 l+1)} h_{l}\left(k r_{p q}^{\prime}\right), \quad(R \mid R)_{l}^{0}\left(r_{p q}^{\prime}\right)=(S \mid S)_{l}^{0}\left(r_{p q}^{\prime}\right)=(-1)^{l} \sqrt{(2 l+1)} j_{l}\left(k r_{p q}^{\prime}\right) \\
& b_{m+1}^{-m-1}(E \mid F)_{l}^{m+1}=b_{l}^{-m-1}(E \mid F)_{l-1}^{m}-b_{l+1}^{m}(E \mid F)_{l+1}^{m}, \quad E, F=S, R, \quad l=m+1, m+2, \ldots,
\end{aligned}
$$

Note that for computation of the reexpansion coef cients inside an $(l, n, m)$ cube of size $\left(N_{t}, N_{t}, N_{t}\right)$, the coef cients $(E \mid F)_{l 0}^{0}$ must be computed for $l=0, \ldots, 2 N_{t}$. This is because the recurrence relations for increase of $n(4.79)$ and for increase of $l$ (4.86) require knowledge of $(E \mid F)_{l+1, n}^{m}$ to compute $(E \mid F)_{l, n+1}^{m}$ and $(E \mid F)_{l n}^{m+1}$. 


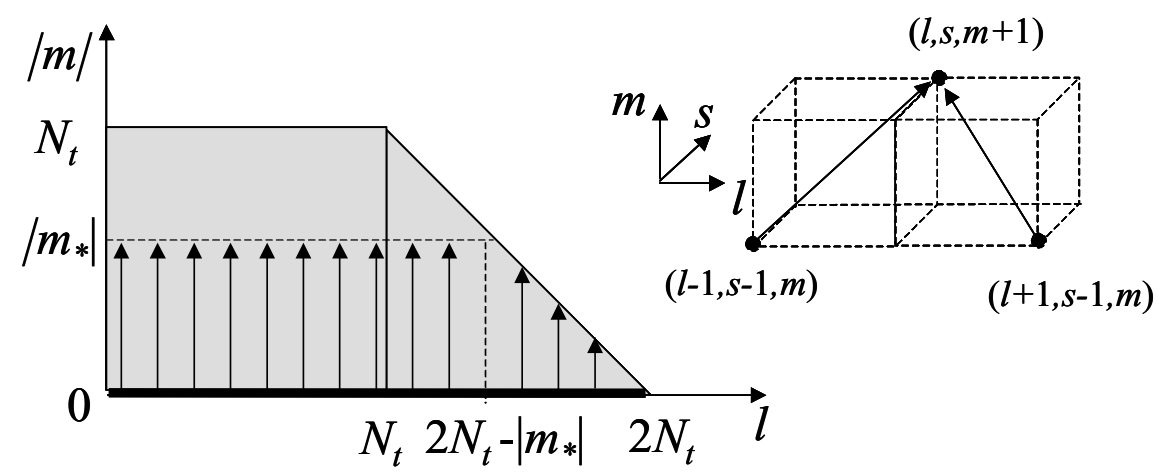

FIG. 4.1. A scheme for computation of the sectorial coef cients. Schematic of relation (4.67), which enables propagation in $(l, s, m)$-space for increasing $m$ is shown on the right. Schematic of lling a block of coef cients in this space from initial values speci ed at $m=0$ is shown on the left (in projection to the $(l, m)$-plane).

4.9. Computation of Translation Coef cients. A variety of recurrence relations provide various strategies for computation of translation coef cients $(E \mid F)_{l n}^{s m}\left(\mathbf{r}_{p q}^{\prime}\right)$ in a speci ed range of indices $n, l, m$, and $s$. Below we represent one of the possible algorithms, which we used for computation of the $S \mid R$-translation matrix in multiple scattering problem [24]. In this problem we needed a truncated matrix, where the indices lie in the range $-N_{t} \leqslant m, s \leqslant N_{t}$, $0 \leqslant n, l \leqslant N_{t}$, where $N_{t}$ is the truncation number. First, we note that the range of non-zero coef cients is bounded by $m \leqslant n$ and $|s| \leqslant l$.

The process starts with speci cation of initial values $(E \mid F)_{l 0}^{s 0}$ using Eq. (4.43) or (4.58) for $l=0, \ldots, 2 N_{t}$, and $s=-l, \ldots, l$. Eq. (4.67) shows that these data provide computation of the sectorial coef cients $(E \mid F)_{l,}^{s m}$ at $m=1$ for $l=0, \ldots, 2 N_{t}-1$ and $s=-l, \ldots, l$ and further till $m=N_{t}$, where the range $l=0, \ldots, N_{t}, s=-l, \ldots, l$ is covered. Similarly, Eq. (4.68) enables computation of the sectorial coef cients $(E \mid F)_{l}^{s m}$ for $m=-1, \ldots,-N_{t}$ and the same range of $l$ and $s$. Symmetry (4.61) is used to nd sectorial coef cients $(E \mid F)_{, n}^{s m}$ for $s=-N_{t}, \ldots, N_{t}$ and $n=0, \ldots, 2 N_{t}-|s|, m=-n, \ldots, n$. A scheme for computation of the sectorial translation coef cients is shown in Fig. 4.1.

Consider now computation of other coef cients. For this purpose we use Eq. (4.26) at the layer $m=$ const, $s=$ const. Assume that $|s| \leqslant|m|$. For such a layer we have coef cients known at $n=|m|$ and $l=|s|, \ldots, 2 N_{t}-|m|$. At $n=|m|+1$ Eq. (4.26) yield values of the translation coef cients for $l=|s|, \ldots, 2 N_{t}-|m|-1$ and further, until $n=N_{t}$ and $l=|s|, \ldots, N_{t}$. This lls some trapezoidal domain in the $(n, l)$-plane from left to right (see Fig. 4.2). The rest of the domain required for lling is performed by applying the same recurrence relation, but with lling from the bottom to the top by propagation with respect to $l$. So we use known values of the sectorial coef cients at $l=|s|$ and $n=|m|, \ldots, 2 N_{t}-|s|$ and use recursion (4.26) resolved with respect to coef cients for $l+1$. A similar procedure holds for $|m| \leqslant|s|$ (see Fig. 4.2). So this algorithm enables computation of all translation coef cients inside the speci ed domain.

4.9.1. Number of Operations. THEOREM 4.10. Computation of multipole reexpansion, or translation, coef cients $(E \mid F)_{l n}^{s m}\left(\mathbf{r}_{p q}\right)$ for all values of $l=0, \ldots, N_{t}, s=-l, \ldots, l, n=0, \ldots, N_{t}, m=-n, \ldots, n$ can be performed within $O\left(N_{t}^{4}\right)$ operations.

Proof. The total number of coef cients $(E \mid F)_{l n}^{s m}, l=0, \ldots, N_{t}, s=-l, \ldots, l, n=0, \ldots, N_{t}, m=-n, \ldots, n$, is $\left(N_{t}+1\right)^{4}=O\left(N_{t}^{4}\right)$. So, even if each coef cient can be computed in constant time the number of operations will be bounded from below by $O\left(N_{t}^{4}\right)$. Computation of the initial values $(E \mid F)_{l 0}^{s 0}$ and $(E \mid F)_{0 n}^{0 m}$ requires $O\left(N_{t}\right)$ operations. Computation of the sectorial coef cients $(E \mid F)_{l|m|}^{s m}$ and $(E \mid F)_{|s| n}^{s m}$ using the initial values and recurrence relations, which include not more than 2 multiplications and one addition to produce a new value requires $O\left(N_{t}^{3}\right)$ operations, since the total number of the sectorial coef cients is $O\left(N_{t}^{3}\right)$. Computation of the tesseral coef cients $(E \mid F)_{l n}^{s m}$ using the values of the sectorial coef cients recurrence relations which include not more than 3 multiplications and 2 additions requires $O\left(N_{t}^{4}\right)$ operations. In the recurrence process additional values of $(E \mid F)_{l n}^{s m}$ for $l>N_{t}$ and $n>N_{t}$ may be required. However the maximum size of $l$ and $n$ is limited by $2 N_{t}$. Thus the number of operations is $O\left(N_{t}^{4}\right)$.

THEOREM 4.11. Computation of coaxial multipole reexpansion, or translation, coef cients $(E \mid F)_{l n}^{m}\left(r_{p q}\right)$ for all values of $l=0, \ldots, N_{t}, n=0, \ldots, N_{t}, m=-n, \ldots, n$ can be performed in $O\left(N_{t}^{3}\right)$ operations. 

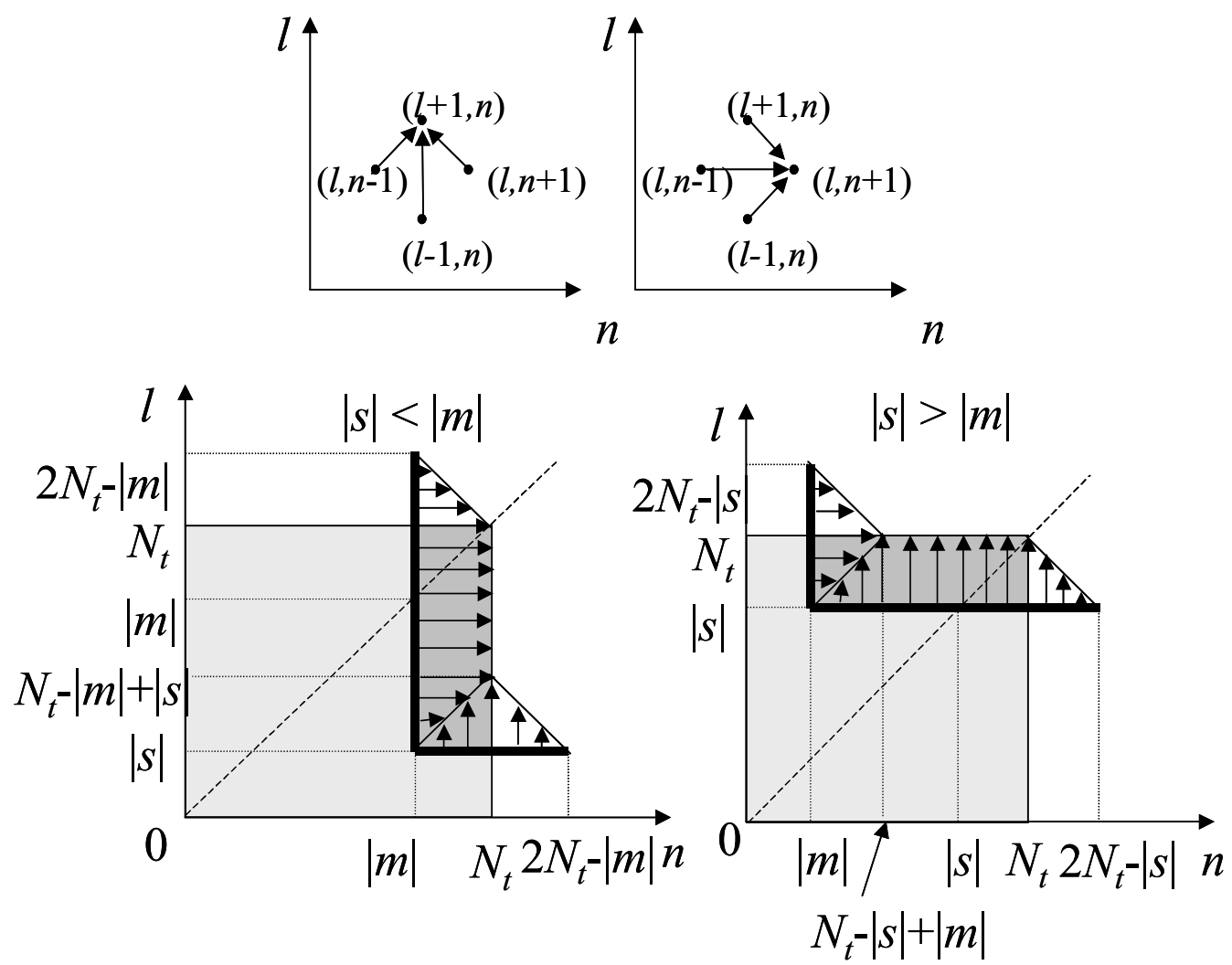

FIG. 4.2. A scheme for recursive computation of the translation coef cients at layer $m=$ const, $s=$ const. Propagation according Eq. (4.26) can be performed with respect to $l$ or to $n$ as shown in the top graphs. Using precomputed sectorial coef cients at $l=|s|$ and $n=|m|$ and these propagation schemes one can compute all translation coef cients in the speci ed range, $|s| \leqslant l \leqslant N_{t},|m| \leqslant n \leqslant N_{t}$. To reduce the amount of unnecessary computations two cases, $|s| \leqslant|m|$ and $|m|<|s|$ can be considered as shown in gures on the bottom right and left.

Proof. The proof follows similarly.

\section{Rotation Coef cients.}

5.1. Rotation Matrix. It is often convenient (and physically meaningful) to link the entries of the matrix $Q 2.24$ to physical angles between the axes. This is usually done via one of many representations such as the Euler angles. In the present case we wish to rotate a set of axes so that the old $z$ axis is rotated to a speci ed $\hat{z}$ direction (pointing to the translation location of the multipole). A simple expression for $Q$ in terms of the direction cosines of the $\hat{z}$ direction that achieves this objective can be derived from elementary geometric considerations. We recall from Euler's theorem [20] that any rotation of a rigid body can be uniquely speci ed by providing an axis of rotation and the angle of rotation through that axis.

Referring to Figure 5.1, the origin and the two $z$ axes form a given plane $(O z \hat{z})$. In this case the vector that is normal to this plane, and passes through the origin is obviously the axis of rotation. Let the direction cosines of the new $\hat{z}$ axis be $e_{x}, e_{y}, e_{z}$, and let the direction of the $z$ axis in the current coordinate system be $\mathbf{i}_{z}$. Then the angle $\gamma$ through which we must rotate the original system about the rotation axis is speci ed by

$$
\cos \gamma=e_{z}
$$

The direction of the axis of rotation can be speci ed as

$$
\mathbf{n}=\mathbf{i}_{z} \times \mathbf{i}_{\bar{z}}=\left|\begin{array}{ccc}
\mathbf{i}_{x} & \mathbf{i}_{y} & \mathbf{i}_{z} \\
0 & 0 & 1 \\
e_{x} & e_{y} & e_{z}
\end{array}\right|=-e_{y} \mathbf{i}_{x}+e_{x} \mathbf{i}_{y} .
$$




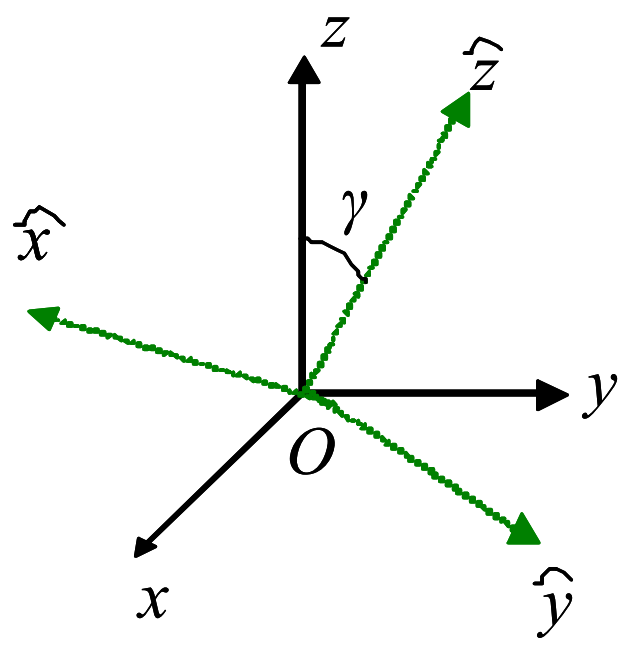

FIG. 5.1. Rotation of axes.

Let us make a choice that the new $\mathbf{i}_{\widehat{x}}$ direction is along $\mathbf{n}$. The unit vector along this direction is

$$
\mathbf{i}_{\widehat{x}}=\frac{-e_{y} \mathbf{i}_{x}+e_{x} \mathbf{i}_{y}}{\sqrt{\left(e_{x}^{2}+e_{y}^{2}\right)}}
$$

We then have the remaining axis chosen by the cyclic order of coordinate vectors as

$$
\mathbf{i}_{\widehat{y}}=\mathbf{i}_{\widehat{z}} \times \mathbf{i}_{\widehat{x}}=\frac{1}{\sqrt{\left(e_{x}^{2}+e_{y}^{2}\right)}}\left|\begin{array}{ccc}
\mathbf{i}_{x} & \mathbf{i}_{y} & \mathbf{i}_{z} \\
e_{x} & e_{y} & e_{z} \\
-e_{y} & e_{x} & 0
\end{array}\right|=\frac{-e_{z} e_{x} \mathbf{i}_{x}-e_{z} e_{y} \mathbf{i}_{y}}{\sqrt{\left(e_{x}^{2}+e_{y}^{2}\right)}}+\sqrt{\left(e_{x}^{2}+e_{y}^{2}\right)} \mathbf{i}_{z}
$$

We can now evaluate the matrix $Q$ using Equation (2.24) as

$$
Q=\left[\begin{array}{ccc}
\mathbf{i}_{\widehat{x}} \cdot \mathbf{i}_{x} & \mathbf{i}_{\widehat{x}} \cdot \mathbf{i}_{y} & \mathbf{i}_{\widehat{x}} \cdot \mathbf{i}_{z} \\
\mathbf{i}_{\widehat{y}} \cdot \mathbf{i}_{x} & \mathbf{i}_{\widehat{y}} \cdot \mathbf{i}_{y} & \mathbf{i}_{\widehat{y}} \cdot \mathbf{i}_{z} \\
\mathbf{i}_{\widehat{z}} \cdot \mathbf{i}_{x} & \mathbf{i}_{\widehat{z}} \cdot \mathbf{i}_{y} & \mathbf{i}_{\widehat{z}} \cdot \mathbf{i}_{z}
\end{array}\right]=\left[\begin{array}{ccc}
-\frac{e_{y}}{\sqrt{\left(e_{x}^{2}+e_{y}^{2}\right)}} & \frac{e_{x}}{\sqrt{\left(e_{x}^{2}+e_{y}^{2}\right)}} & 0 \\
-\frac{e_{z} e_{x}}{\sqrt{\left(e_{x}^{2}+e_{y}^{2}\right)}} & -\frac{e_{z} e_{y}}{\sqrt{\left(e_{x}^{2}+e_{y}^{2}\right)}} & \sqrt{\left(e_{x}^{2}+e_{y}^{2}\right)} \\
e_{x} & e_{y} & e_{z}
\end{array}\right] .
$$

Of course, here the choice of the $\hat{x}$ and the $\hat{y}$ axes was arbitrary. If we have a speci cation for the orientation of these axes (thereby xing the $0 b 0$ meridian in the rotated coordinate system), we can compute the $Q$ matrix as a composition of two rotations as

$$
Q=\left[\begin{array}{ccc}
\cos \phi & -\sin \phi & 0 \\
\sin \phi & \cos \phi & 0 \\
0 & 0 & 1
\end{array}\right]\left[\begin{array}{ccc}
-\frac{e_{y}}{\sqrt{\left(e_{x}^{2}+e_{y}^{2}\right)}} & \frac{e_{x}}{\sqrt{\left(e_{x}^{2}+e_{y}^{2}\right)}} & 0 \\
-\frac{e_{z} e_{x}}{\sqrt{\left(e_{x}^{2}+e_{y}^{2}\right)}} & -\frac{e_{z} e_{y}}{\sqrt{\left(e_{x}^{2}+e_{y}^{2}\right)}} & \sqrt{\left(e_{x}^{2}+e_{y}^{2}\right)} \\
e_{x} & e_{y} & e_{z}
\end{array}\right] .
$$

where $\phi$ is the rotation angle near $\mathbf{i}_{z}$ axis.

For computation of rotations of spherical harmonics it is convenient to represent the rotation matrix using spherical polar angles in both coordinate systems $\left(\mathbf{i}_{x}, \mathbf{i}_{y}, \mathbf{i}_{z}\right)$ and $\left(\mathbf{i}_{\widehat{x}}, \mathbf{i}_{\widehat{y}}, \mathbf{i}_{\widehat{z}}\right)$. Let $\theta^{\prime}$ and $\varphi^{\prime}$ be the spherical angles of the axis $\mathbf{i}_{z}$ in the reference frame $\left(\mathbf{i}_{\hat{x}}, \mathbf{i}_{\widehat{y}}, \mathbf{i}_{z}\right)$, and let $\gamma$ and $\chi$ be the spherical angles of the axis $\mathbf{i}_{z}$ in the reference frame $\left(\mathbf{i}_{x}, \mathbf{i}_{y}, \mathbf{i}_{z}\right)$. The angles $\theta^{\prime}$ and $\gamma$ are the same since $\cos \theta^{\prime}=\mathbf{i}_{z} \cdot \mathbf{i}_{\bar{z}}=\mathbf{i}_{\bar{z}} \cdot \mathbf{i}_{z}=\cos \gamma=e_{z}$. The three independent angles $\theta^{\prime}, \varphi^{\prime}$, and $\chi$ uniquely specify arbitrary rotation. 

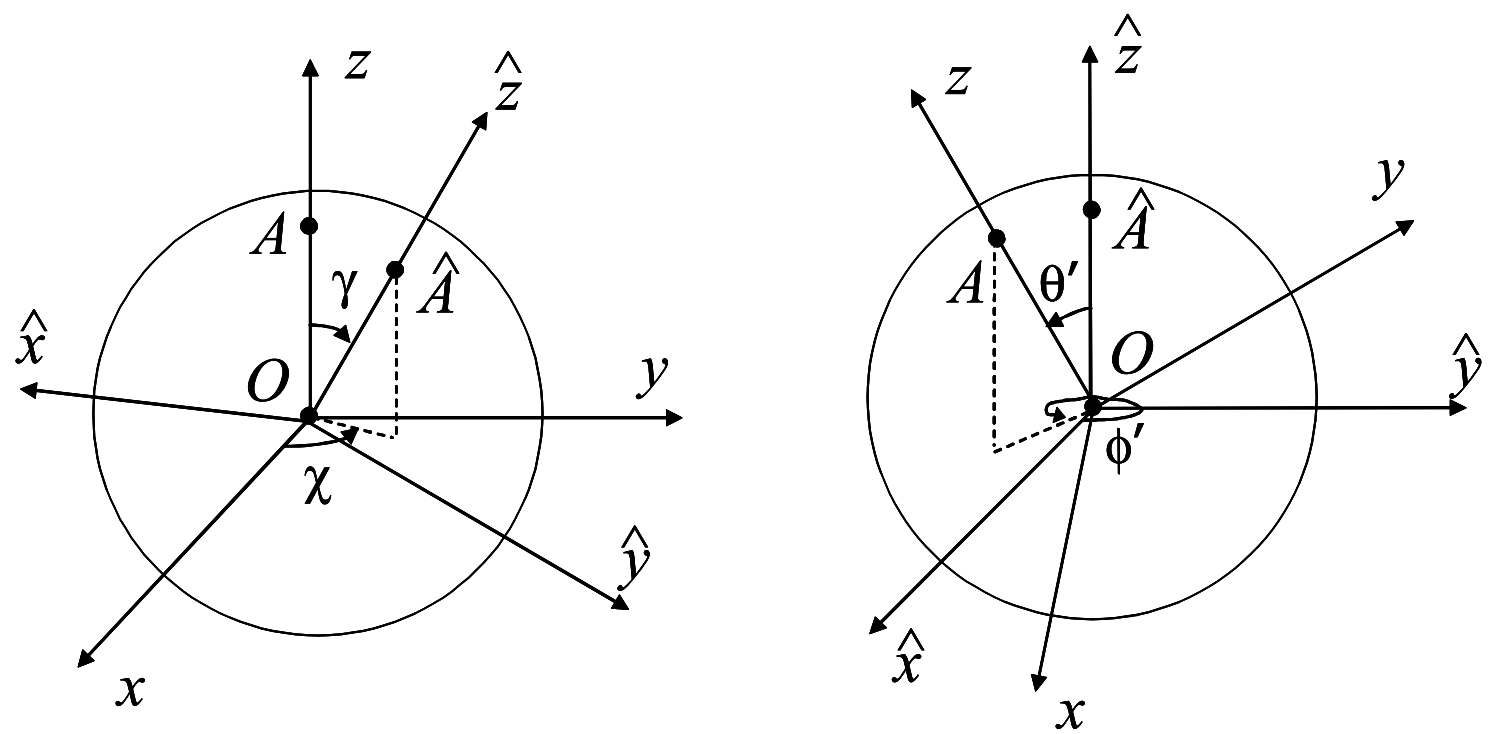

FIG. 5.2. The gure on the left shows the transformed axes $(\hat{x}, \hat{y}, \hat{z})$ in the original reference frame $(x, y, z)$. The spherical polar coordinates of the point $\hat{A}$ lying on the $\hat{z}$ axis on the unit sphere are $(\gamma, \chi)$. The gure on the right shows the original axes $(x, y, z)$ in the transformed reference frame $(\hat{x}, \hat{y}, \hat{z})$. The coordinates of the point A lying on the $z$ axis on the unit sphere are $\left(\theta^{\prime}=\gamma, \varphi^{\prime}\right)$. The points $O, A$, and $\hat{A}$ are the same in both gures. All rotation matrices can be derived in terms of these three angles $\theta^{\prime}, \varphi^{\prime}, \chi$. Particularly economical expressions for multipole rotations can be obtained using these angles, as shown in the text.

The relation between the components of the rotation matrix (5.4) and angles $\theta^{\prime}$ and $\varphi^{\prime}$ is provided by the following:

$$
\begin{aligned}
& \cos \theta^{\prime}=\mathbf{i}_{z} \cdot \mathbf{i}_{\widehat{z}}=Q_{33}=e_{z}, \quad \theta^{\prime}=\gamma . \\
& \cos \varphi^{\prime} \sin \theta^{\prime}=\mathbf{i}_{z} \cdot \mathbf{i}_{\widehat{x}}=Q_{13}=-\sqrt{\left(e_{x}^{2}+e_{y}^{2}\right)} \sin \phi, \\
& \sin \varphi^{\prime} \sin \theta^{\prime}=\mathbf{i}_{z} \cdot \mathbf{i}_{\widehat{y}}=Q_{23}=\sqrt{\left(e_{x}^{2}+e_{y}^{2}\right)} \cos \phi .
\end{aligned}
$$

Thus, the rotation angle $\phi$ and the polar angle $\varphi^{\prime}$ are related as

$$
\phi=\varphi^{\prime}-\frac{\pi}{2}
$$

At the same time we have

$$
\begin{aligned}
& e_{x}=\mathbf{i}_{\widehat{z}} \cdot \mathbf{i}_{x}=\sin \gamma \cos \chi=\sin \theta^{\prime} \cos \chi \\
& e_{y}=\mathbf{i}_{\widehat{z}} \cdot \mathbf{i}_{y}=\sin \gamma \sin \chi=\sin \theta^{\prime} \sin \chi
\end{aligned}
$$

The matrix $Q$ representing the rotation between the axes can be represented in terms of these angles in the form

$$
\begin{aligned}
Q\left(\theta^{\prime}, \varphi^{\prime}, \chi\right) & =\left[\begin{array}{ccc}
\sin \varphi^{\prime} & \cos \varphi^{\prime} & 0 \\
-\cos \varphi^{\prime} & \sin \varphi^{\prime} & 0 \\
0 & 0 & 1
\end{array}\right]\left[\begin{array}{ccc}
-\sin \chi & \cos \chi & 0 \\
-\cos \theta^{\prime} \cos \chi & -\cos \theta^{\prime} \sin \chi & \sin \theta^{\prime} \\
\sin \theta^{\prime} \cos \chi & \sin \theta^{\prime} \sin \chi & \cos \theta^{\prime}
\end{array}\right] \\
& =\left[\begin{array}{ccc}
-\sin \varphi^{\prime} \sin \chi-\cos \theta^{\prime} \cos \varphi^{\prime} \cos \chi & \sin \varphi^{\prime} \cos \chi-\cos \theta^{\prime} \cos \varphi^{\prime} \sin \chi & \sin \theta^{\prime} \cos \varphi^{\prime} \\
\cos \varphi^{\prime} \sin \chi-\cos \theta^{\prime} \sin \varphi^{\prime} \cos \chi & -\left(\cos \varphi^{\prime} \cos \chi+\cos \theta^{\prime} \sin \varphi^{\prime} \sin \chi\right) & \sin \theta^{\prime} \sin \varphi^{\prime} \\
\sin \theta^{\prime} \cos \chi & \sin \theta^{\prime} \sin \chi & \cos \theta^{\prime}
\end{array}\right] .
\end{aligned}
$$

Since $Q$ is an orthogonal rotation matrix, it satis es

$$
\left[Q\left(\theta^{\prime}, \varphi^{\prime}, \chi\right)\right]^{-1}=\left[Q\left(\theta^{\prime}, \varphi^{\prime}, \chi\right)\right]^{T}=Q\left(\theta^{\prime}, \chi, \varphi^{\prime}\right) .
$$


The last equality holds because we can exchange the angles $\chi$ and $\varphi^{\prime}$ if we exchange the order of the basis vectors $\left(\mathbf{i}_{x}, \mathbf{i}_{y}, \mathbf{i}_{z}\right)$ and $\left(\mathbf{i}_{\widehat{x}}, \mathbf{i}_{\widehat{y}}, \mathbf{i}_{\widehat{z}}\right)$. Note also that $Q$ can be represented as a composition of three rotations:

$Q\left(\theta^{\prime}, \varphi^{\prime}, \chi\right)=\left[\begin{array}{ccc}\sin \varphi^{\prime} & \cos \varphi^{\prime} & 0 \\ -\cos \varphi^{\prime} & \sin \varphi^{\prime} & 0 \\ 0 & 0 & 1\end{array}\right]\left[\begin{array}{ccc}-1 & 0 & 0 \\ 0 & -\cos \theta^{\prime} & \sin \theta^{\prime} \\ 0 & \sin \theta^{\prime} & \cos \theta^{\prime}\end{array}\right]\left[\begin{array}{ccc}\sin \chi & -\cos \chi & 0 \\ \cos \chi & \sin \chi & 0 \\ 0 & 0 & 1\end{array}\right]=A\left(\varphi^{\prime}\right) B\left(\theta^{\prime}\right) A^{T}(\chi)$

Here $B\left(\theta^{\prime}\right)=\left[B\left(\theta^{\prime}\right)\right]^{-1}=\left[B\left(\theta^{\prime}\right)\right]^{T}$ and

$\left[Q\left(\theta^{\prime}, \varphi^{\prime}, \chi\right)\right]^{-1}=\left[A\left(\varphi^{\prime}\right) B\left(\theta^{\prime}\right) A^{T}(\chi)\right]^{-1}=\left[A^{T}(\chi)\right]^{-1}\left[B\left(\theta^{\prime}\right)\right]^{-1}\left[A\left(\varphi^{\prime}\right)\right]^{-1}=A(\chi) B\left(\theta^{\prime}\right) A^{T}\left(\varphi^{\prime}\right)=Q\left(\theta^{\prime}, \chi, \varphi^{\prime}\right)$.

Such a symmetrical representation using the angles $\theta^{\prime}, \varphi^{\prime}$, and $\chi$ leads to compact expressions for the spherical coordinates of vectors in the rotated reference frame.

Note that the angles $\left(\varphi^{\prime}, \theta^{\prime}, \chi\right)$ are simply related to the standard Euler rotation angles (denote them $\alpha_{E}, \beta_{E}, \gamma_{E}$ to avoid confusion with previously de ned angles):

$$
\alpha_{E}=\pi-\chi, \quad \beta_{E}=\theta^{\prime}, \quad \gamma_{E}=\varphi^{\prime} .
$$

This can be checked in a straightforward way by comparing the representations of the rotation matrix $Q$ in the form (5.8) and via the Euler angles which can be found elsewhere (e.g., Ref. [20]).

5.2. Integral Representation of Rotation Coef cients. Before considering the ef cient evaluation of the rotation coef cients $T_{n}^{\nu m}(Q)$, we consider their evaluation using their de nition (2.25) and the fact that spherical harmonics (2.5) form a complete orthonormal system. Using the de nition of the scalar product (2.2) we obtain:

$$
T_{n}^{\nu m}(Q)=\left(Y_{n}^{m}(\theta, \varphi), Y_{n}^{\nu}(\widehat{\theta}, \widehat{\varphi})\right)=\int_{-\pi}^{\pi} d \widehat{\varphi} \int_{0}^{\pi} Y_{n}^{m}(\theta, \varphi) Y_{n}^{-\nu}(\widehat{\theta}, \widehat{\varphi}) \sin \widehat{\theta} \widehat{d} \widehat{\theta}
$$

5.3. Structure of the Rotation Coef cients. The structure of the rotation coef cients can be found from the properties of the rotation matrix. Since the rotation matrix can be decomposed to three rotations about the axes with angles $\chi, \theta^{\prime}$, and $\varphi^{\prime}$ we can see how spherical harmonics change with such a transform. First we note that rotation of coordinates with the angles $\chi$ and $\varphi^{\prime}$ do not change quantities that depend on $\theta^{\prime}$, or $\gamma$. For example, the $\chi$-rotation conserves the original $z$ axis and denoting the angle $\varphi$ in the original coordinates as $\varphi_{1}$ in the rotated coordinates, we have

$$
\varphi=\varphi_{1}+\chi
$$

The spherical harmonics then change as

$$
Y_{n}^{m}(\theta, \varphi)=Y_{n}^{m}\left(\theta, \varphi_{1}+\chi\right)=e^{i m \chi} Y_{n}^{m}\left(\theta, \varphi_{1}\right) .
$$

The transform of the spherical harmonics from the $\chi$-rotated coordinates to the nal coordinates can be described by:

$$
Y_{n}^{m}\left(\theta, \varphi_{1}\right)=\sum_{\nu=-n}^{n} T_{n}^{(1) \nu m}\left(\theta^{\prime}, \varphi^{\prime}\right) Y_{n}^{\nu}(\widehat{\theta}, \widehat{\varphi})
$$

When we perform the last rotation of coordinates to arrive at the angle $\phi=\varphi^{\prime}-\frac{\pi}{2}$, we have the same situation, and so

$$
\widehat{\varphi}=\varphi_{2}+\phi=\varphi_{2}+\varphi^{\prime}-\frac{\pi}{2},
$$

where $\varphi_{2}$ is the angle corresponding to the given point in coordinates where the last rotation was not performed. So

$$
Y_{n}^{\nu}(\widehat{\theta}, \widehat{\varphi})=Y_{n}^{\nu}\left(\widehat{\theta}, \varphi_{2}+\varphi^{\prime}-\frac{\pi}{2}\right)=(-i)^{\nu} e^{i \nu \varphi^{\prime}} Y_{n}^{\nu}\left(\widehat{\theta}, \varphi_{2}\right) .
$$


The transform from $Y_{n}^{m}\left(\theta, \varphi_{1}\right)$ to $Y_{n}^{m}\left(\widehat{\theta}, \varphi_{2}\right)$ occurs only because of the rotation related to angle $\theta^{\prime}$, and so

$$
Y_{n}^{m}\left(\theta, \varphi_{1}\right)=\sum_{\nu=-n}^{n} T_{n}^{(12) \nu m}\left(\theta^{\prime}\right) Y_{n}^{\nu}\left(\widehat{\theta}, \varphi_{2}\right)
$$

Combining these results we nd that:

$$
Y_{n}^{m}(\theta, \varphi)=e^{i m \chi} \sum_{\nu=-n}^{n} i^{\nu} e^{-i \nu \varphi^{\prime}} T_{n}^{(12) \nu m}\left(\theta^{\prime}\right) Y_{n}^{\nu}(\widehat{\theta}, \widehat{\varphi})
$$

This shows that

$$
T_{n}^{\nu m}\left(\theta^{\prime}, \varphi^{\prime}, \chi\right)=e^{i m \chi} e^{-i \nu \varphi^{\prime}} H_{n}^{\nu m}\left(\theta^{\prime}\right), \quad H_{n}^{\nu m}\left(\theta^{\prime}\right)=i^{\nu} T_{n}^{(12) \nu m}\left(\theta^{\prime}\right) .
$$

An explicit expression for $H_{n}^{\nu m}\left(\theta^{\prime}\right)$ can be found in the paper of Stein [15], which in the notation of the present paper $^{1}$ can be represented in the form

$$
H_{n}^{\nu m}\left(\theta^{\prime}\right)=\epsilon_{m} \epsilon_{\nu}[(n+\nu) !(n-\nu) !(n+m) !(n-m) !]^{1 / 2} \sum_{\sigma=\max (0,-(m+\nu))}^{\min (n-m, n-\nu)} \frac{(-1)^{n-\sigma} \cos ^{2 \sigma+\nu+m} \frac{1}{2} \theta^{\prime} \sin ^{2 n-2 \sigma-\nu-m} \frac{1}{2} \theta^{\prime}}{\sigma !(n-m-\sigma) !(n-\nu-\sigma) !(m+\nu+\sigma) !} .
$$

5.4. Recurrence Relations for Rotation Coef cients. THEOREM 5.1. The following recurrence relations holds for $T_{n}^{\nu m}(Q)$ :

$$
\begin{aligned}
\frac{1}{2}\left(\mathbf{i}_{x}-i \mathbf{i}_{y}\right) b_{n+1}^{-m-1} T_{n+1}^{\nu, m+1}+\frac{1}{2}\left(\mathbf{i}_{x}+i \mathbf{i}_{y}\right) b_{n+1}^{m-1} T_{n+1}^{\nu, m-1}-\mathbf{i}_{z} a_{n}^{m} T_{n+1}^{\nu m} & =\frac{1}{2}\left(\mathbf{i}_{\hat{x}}-i \mathbf{i}_{\hat{y}}\right) b_{n+1}^{-\nu} T_{n}^{\nu-1, m} \\
& +\frac{1}{2}\left(\mathbf{i}_{\hat{x}}+i \mathbf{i}_{\hat{y}}\right) b_{n+1}^{\nu} T_{n}^{\nu+1, m}-\mathbf{i}_{\hat{z}} a_{n}^{\nu} T_{n}^{\nu m}
\end{aligned}
$$

where $n=0,1,2, \ldots, m=-n, \ldots, n, \nu=-n-1, \ldots, n+1$, and

$$
\begin{aligned}
\frac{1}{2}\left(\mathbf{i}_{x}-i \mathbf{i}_{y}\right) b_{n}^{m} T_{n-1}^{\nu, m+1}+\frac{1}{2}\left(\mathbf{i}_{x}+i \mathbf{i}_{y}\right) b_{n}^{-m} T_{n-1}^{\nu, m-1}-\mathbf{i}_{z} a_{n-1}^{m} T_{n-1}^{\nu m} & =\frac{1}{2}\left(\mathbf{i}_{\hat{x}}-i \mathbf{i}_{\hat{y}}\right) b_{n}^{\nu-1} T_{n}^{\nu-1, m} \\
& +\frac{1}{2}\left(\mathbf{i}_{\hat{x}}+i \mathbf{i}_{\hat{y}}\right) b_{n}^{-\nu-1} T_{n}^{\nu+1, m}-\mathbf{i}_{\hat{z}} a_{n-1}^{\nu} T_{n}^{\nu m} .
\end{aligned}
$$

where $n=0,1,2, \ldots, m=-n, \ldots, n, \nu=-n+1, \ldots, n-1$.

Proof. Applying the operator $k^{-1} \nabla$ to any of the relations (2.26), we obtain

$$
k^{-1} \nabla F_{n}^{m}\left(\mathbf{r}_{p}\right)=\sum_{\nu=-n}^{n} T_{n}^{\nu m}(Q) k^{-1} \nabla F_{n}^{\nu}\left(\widehat{\mathbf{r}}_{p}\right), \quad\left|\widehat{\mathbf{r}}_{p}\right|=\left|\mathbf{r}_{p}\right|, \quad F=S, R
$$

The operator $\nabla$ is independent of the reference frame it is represented in. This applies to rotations as well, and so $\nabla=\widehat{\nabla}$. We can use Equation (3.7), to represent the left and right hand sides of (5.23). Grouping terms corresponding to the same basis functions $F_{n}^{\nu}\left(\widehat{\mathbf{r}}_{p}\right)$ we obtain the statement of the theorem. A more detailed proof is provided in Gumerov and Duraiswami [2].

THEOREM 5.2. The following recurrence relations holds for $T_{n}^{\nu m}(Q)$ :

$$
\begin{aligned}
b_{n+1}^{-m-1} T_{n+1}^{\nu, m+1}+b_{n+1}^{m-1} T_{n+1}^{\nu, m-1} & =W_{11} b_{n+1}^{-\nu} T_{n}^{\nu-1, m}+W_{12} b_{n+1}^{\nu} T_{n}^{\nu+1, m}+W_{13} a_{n}^{\nu} T_{n}^{\nu m}, \\
b_{n+1}^{-m-1} T_{n+1}^{\nu, m+1}-b_{n+1}^{m-1} T_{n+1}^{\nu, m-1} & =W_{21} b_{n+1}^{-\nu} T_{n}^{\nu-1, m}+W_{22} b_{n+1}^{\nu} T_{n}^{\nu+1, m}+W_{23} a_{n}^{\nu} T_{n}^{\nu m} \\
a_{n}^{m} T_{n+1}^{\nu m} & =W_{31} b_{n+1}^{-\nu} T_{n}^{\nu-1, m}+W_{32} b_{n+1}^{\nu} T_{n}^{\nu+1, m}+W_{33} a_{n}^{\nu} T_{n}^{\nu m}
\end{aligned}
$$

\footnotetext{
${ }^{1}$ Note that in [15] the Euler angles are de ned with sign opposite to our de nitions of $\alpha_{E}, \beta_{E}$, and $\gamma_{E}$.
} 
where $n=0,1,2, \ldots, m=-n, \ldots, n, \nu=-n-1, \ldots, n+1$, and $W_{\alpha \beta}$ are the elements of the following complex rotation matrix

$$
\mathbf{W}=\left(\begin{array}{ccc}
\mathbf{i}_{x} \cdot\left(\mathbf{i}_{\hat{x}}-i \mathbf{i}_{\hat{y}}\right) & \mathbf{i}_{x} \cdot\left(\mathbf{i}_{\hat{x}}+i \mathbf{i}_{\hat{y}}\right) & -2 \mathbf{i}_{x} \cdot \mathbf{i}_{\hat{z}} \\
i \mathbf{i}_{y} \cdot\left(\mathbf{i}_{\hat{x}}-i \mathbf{i}_{\hat{y}}\right) & i \mathbf{i}_{y} \cdot\left(\mathbf{i}_{\hat{x}}+i \mathbf{i}_{\hat{y}}\right) & -2 i \mathbf{i}_{y} \cdot \mathbf{i}_{\hat{z}} \\
-\frac{1}{2} \mathbf{i}_{z} \cdot\left(\mathbf{i}_{\hat{x}}-i \mathbf{i}_{\hat{y}}\right) & -\frac{1}{2} \mathbf{i}_{z} \cdot\left(\mathbf{i}_{\hat{x}}+i \mathbf{i}_{\hat{y}}\right) & \mathbf{i}_{z} \cdot \mathbf{i}_{\hat{z}}
\end{array}\right)
$$

Proof. Taking scalar product with $\mathbf{i}_{x}, i \mathbf{i}_{y}$, and $\mathbf{i}_{z}$ of both sides of (5.21), we obtain the theorem statement. COROLLARY 5.3. Summing and subtracting relations (5.24) and (5.25) we have

$$
\begin{aligned}
2 b_{n+1}^{-m-1} T_{n+1}^{\nu, m+1} & =\left(W_{11}+W_{21}\right) b_{n+1}^{-\nu} T_{n}^{\nu-1, m}+\left(W_{12}+W_{22}\right) b_{n+1}^{\nu} T_{n}^{\nu+1, m}+\left(W_{13}+W_{23}\right) a_{n}^{\nu} T_{n}^{\nu m}, \\
2 b_{n+1}^{m-1} T_{n+1}^{\nu, m-1} & =\left(W_{11}-W_{21}\right) b_{n+1}^{-\nu} T_{n}^{\nu-1, m}+\left(W_{12}-W_{22}\right) b_{n+1}^{\nu} T_{n}^{\nu+1, m}+\left(W_{13}-W_{23}\right) a_{n}^{\nu} T_{n}^{\nu m}
\end{aligned}
$$

For $\nu=n+1$ we have

$$
\begin{aligned}
2 b_{n+1}^{-m-1} T_{n+1}^{n+1, m+1} & =\left(W_{11}+W_{21}\right) b_{n+1}^{-n-1} T_{n}^{n m} \\
2 b_{n+1}^{m-1} T_{n+1}^{n+1, m-1} & =\left(W_{11}-W_{21}\right) b_{n+1}^{-n-1} T_{n}^{n m} \\
a_{n}^{m} T_{n+1}^{n+1, m} & =W_{31} b_{n+1}^{-n-1} T_{n}^{n m}
\end{aligned}
$$

For $\nu=-n-1$ we have

$$
\begin{aligned}
2 b_{n+1}^{-m-1} T_{n+1}^{-n-1, m+1} & =\left(W_{12}+W_{22}\right) b_{n+1}^{-n-1} T_{n}^{-n, m}, \\
2 b_{n+1}^{m-1} T_{n+1}^{-n-1, m-1} & =\left(W_{12}-W_{22}\right) b_{n+1}^{-n-1} T_{n}^{-n, m}, \\
a_{n}^{m} T_{n+1}^{-n-1, m} & =W_{32} b_{n+1}^{-n-1} T_{n}^{-n, m} .
\end{aligned}
$$

THEOREM 5.4. The following recurrence relations holds for $T_{n}^{\nu m}(Q)$ :

$$
\begin{aligned}
b_{n}^{m} T_{n-1}^{\nu, m+1}+b_{n}^{-m} T_{n-1}^{\nu, m-1} & =W_{11} b_{n}^{\nu-1} T_{n}^{\nu-1, m}+W_{12} b_{n}^{-\nu-1} T_{n}^{\nu+1, m}+W_{13} a_{n-1}^{\nu} T_{n}^{\nu m} \\
b_{n}^{m} T_{n-1}^{\nu, m+1}-b_{n}^{-m} T_{n-1}^{\nu, m-1} & =W_{21} b_{n}^{\nu-1} T_{n}^{\nu-1, m}+W_{22} b_{n}^{-\nu-1} T_{n}^{\nu+1, m}+W_{23} a_{n-1}^{\nu} T_{n}^{\nu m} . \\
a_{n-1}^{m} T_{n-1}^{\nu m} & =W_{31} b_{n}^{\nu-1} T_{n}^{\nu-1, m}+W_{32} b_{n}^{-\nu-1} T_{n}^{\nu+1, m}+W_{33} a_{n-1}^{\nu} T_{n}^{\nu m} .
\end{aligned}
$$

where $n=0,1,2, \ldots, m=-n, \ldots, n, \nu=-n+1, \ldots, n-1$, and $W_{\alpha \beta}$ are components of complex rotation matrix (5.27).

Proof. Taking scalar product of both sides of (5.21) with $\mathbf{i}_{x}, i \mathbf{i}_{y}$, and $\mathbf{i}_{z}$, we obtain the theorem statement.

COROLLARY 5.5. Summing and subtracting relations (5.36) and (5.37) we have

$$
\begin{aligned}
2 b_{n}^{m} T_{n-1}^{\nu, m+1} & =\left(W_{11}+W_{21}\right) b_{n}^{\nu-1} T_{n}^{\nu-1, m}+\left(W_{12}+W_{22}\right) b_{n}^{-\nu-1} T_{n}^{\nu+1, m}+\left(W_{13}+W_{23}\right) a_{n-1}^{\nu} T_{n}^{\nu m}, \\
2 b_{n}^{-m} T_{n-1}^{\nu, m-1} & =\left(W_{11}-W_{21}\right) b_{n}^{\nu-1} T_{n}^{\nu-1, m}+\left(W_{12}-W_{22}\right) b_{n}^{-\nu-1} T_{n}^{\nu+1, m}+\left(W_{13}-W_{23}\right) a_{n-1}^{\nu} T_{n}^{\nu m},
\end{aligned}
$$

For $m=n$ we have

$$
\begin{aligned}
\left(W_{11}+W_{21}\right) b_{n}^{\nu-1} T_{n}^{\nu-1, n}+ & \left(W_{12}+W_{22}\right) b_{n}^{-\nu-1} T_{n}^{\nu+1, n}+\left(W_{13}+W_{23}\right) a_{n-1}^{\nu} T_{n}^{\nu n}=0 \\
& W_{31} b_{n}^{\nu-1} T_{n}^{\nu-1, n}+W_{32} b_{n}^{-\nu-1} T_{n}^{\nu+1, n}+W_{33} a_{n-1}^{\nu} T_{n}^{\nu n}=0 .
\end{aligned}
$$

For $m=-n$ we have

$$
\begin{array}{r}
\left(W_{11}-W_{21}\right) b_{n}^{\nu-1} T_{n}^{\nu-1,-n}+\left(W_{12}-W_{22}\right) b_{n}^{-\nu-1} T_{n}^{\nu+1,-n}+\left(W_{13}-W_{23}\right) a_{n-1}^{\nu} T_{n}^{\nu,-n},=0 \\
W_{31} b_{n}^{\nu-1} T_{n}^{\nu-1,-n}+W_{32} b_{n}^{-\nu-1} T_{n}^{\nu+1,-n}+W_{33} a_{n-1}^{\nu} T_{n}^{\nu,-n}=0
\end{array}
$$


5.5. Computational Procedure. We describe briefly a computational procedure to obtain the coef cients $T_{n}^{\nu m}\left(Q_{p q}^{\prime}\right)$ via recurrence relations. It is noteworthy to remark that these coef cients are not a property of the Helmholtz equation but purely a property of spherical harmonics. Due to their dependence only on the angular part, these results should be the same for the Laplace, Schrödinger, heat etc. equations. The spherical harmonics have been studied in greater depth, than have the translation coef cients for the Helmholtz equation (see e.g. Stein [15] for addition theorems, and explicit relations to Wigner's symbols). However this classical problem is still of interest, and research is still ongoing in the stable and rapid computation of the rotation coef cients based on the recurrence relations for real spherical harmonics [18] and general complex case [19]. Our derivation of the recurrence relations differs from these cited papers and has comparable or superior complexity. ${ }^{2}$

5.5.1. Initial Values. Consider (2.25) for $m=0$ :

$$
Y_{n}^{0}(\theta, \varphi)=\sqrt{\frac{2 n+1}{4 \pi}} P_{n}(\cos \theta)=\sum_{\nu=-n}^{n} T_{n}^{\nu 0}(Q) Y_{n}^{\nu}(\widehat{\theta}, \widehat{\varphi}) .
$$

A well-known addition theorem for spherical harmonics (it is reproduced e.g., in Ref. [4]) yields:

$$
P_{n}(\cos \theta)=\frac{4 \pi}{2 n+1} \sum_{\nu=-n}^{n} Y_{n}^{-\nu}\left(\theta^{\prime}, \varphi^{\prime}\right) Y_{n}^{\nu}(\widehat{\theta}, \widehat{\varphi})
$$

where $\theta$ is the angle between points with spherical coordinates $\left(\theta^{\prime}, \varphi^{\prime}\right)$ and $(\widehat{\theta}, \widehat{\varphi})$ on the unit sphere. Comparing (5.45) and (5.46) we obtain

$$
T_{n}^{\nu 0}(Q)=\sqrt{\frac{4 \pi}{2 n+1}} Y_{n}^{-\nu}\left(\theta^{\prime}, \varphi^{\prime}\right), \quad n=0,1, \ldots, \quad \nu=-n, \ldots, n .
$$

Note that $\theta^{\prime}$ and $\varphi^{\prime}$ are nothing but the spherical polar angles of the axis $\mathbf{i}_{z}$ in the reference frame $\left(\mathbf{i}_{\widehat{x}}, \mathbf{i}_{\widehat{y}}, \mathbf{i}_{\widehat{z}}\right)$ and formulae (5.8) and (5.10) provide explicit expressions for components of $Q$ through these angles. Relations (2.5) and (5.47) also provide the following expression for functions $H_{n}^{\nu m}\left(\theta^{\prime}\right)$ de ned by (5.19):

$$
H_{n}^{\nu 0}\left(\theta^{\prime}\right)=(-1)^{\nu} \sqrt{\frac{(n-|\nu|) !}{(n+|\nu|) !}} P_{n}^{|\nu|}\left(\cos \theta^{\prime}\right), \quad n=0,1, \ldots, \quad \nu=-n, \ldots, n .
$$

5.5.2. Symmetry of Rotation Coef cients. THEOREM 5.6. The following symmetry holds

$$
T_{n}^{-\nu,-m}(Q)=\overline{T_{n}^{\nu m}(Q)}, \quad n=0,1, \ldots, \quad \nu, m=-n, \ldots, n .
$$

Proof. Since $Y_{n}^{-\nu}=\overline{Y_{n}^{\nu}}$ for any $n=0,1, \ldots, \nu=-n, \ldots, n$, we have from (2.25):

$$
Y_{n}^{m}(\theta, \varphi)=\overline{Y_{n}^{-m}(\theta, \varphi)}=\sum_{\nu=-n}^{n} \overline{T_{n}^{\nu,-m}(Q) Y_{n}^{\nu}(\widehat{\theta}, \widehat{\varphi})}=\sum_{\nu=-n}^{n} \overline{T_{n}^{\nu,-m}(Q)} Y_{n}^{-\nu}(\widehat{\theta}, \widehat{\varphi})=\sum_{\nu=-n}^{n} \overline{T_{n}^{-\nu,-m}(Q)} Y_{n}^{\nu}(\widehat{\theta}, \widehat{\varphi}) .
$$

Comparing this result with expansion of $Y_{n}^{m}(\theta, \varphi)(2.25)$ we obtain the statement of the theorem, since $Y_{n}^{\nu}(\widehat{\theta}, \widehat{\varphi})$ is orthonormal and representation (2.25) is unique.

COROLlary 5.7. Substituting (5.19) in (2.24) and taking in account that $H_{n}^{\nu m}\left(\theta^{\prime}\right)$ is real, we have

$$
H_{n}^{\nu m}\left(\theta^{\prime}\right)=H_{n}^{-\nu,-m}\left(\theta^{\prime}\right), \quad n=0,1, \ldots, \quad \nu, m=-n, \ldots, n .
$$

Note also that

$$
H_{n}^{\nu m}\left(\theta^{\prime}\right)=H_{n}^{m \nu}\left(\theta^{\prime}\right), \quad n=0,1, \ldots, \quad \nu, m=-n, \ldots, n .
$$

This follows from (5.20).

\footnotetext{
${ }^{2}$ Care should be taken when comparing our results with those of these papers, as we use different de nitions of the spherical harmonics.
} 
5.5.3. Recursive Computation. Since $T_{n}^{\nu 0}(Q)$ are known explicitly for arbitrary $n=0,1,2, \ldots$ and $v=$ $-n, \ldots, n$, we need perform only one-dimensional recursive propagation for $T_{n}^{\nu m}$ for increasing $m(m>0)$ and decreasing $m(m<0)$. The recurrence for negative $m$ can be dropped due to $T_{n}^{\nu m}$ for such $m$ can be simply found using symmetry relation (5.49). For non-negative $m$ we can use any of the relations (5.28) or (5.39).

For example, using the following relation between the elements of matrices $W(5.27)$ and $Q(2.24)$ :

$$
\begin{aligned}
& W_{11}+W_{21}=Q_{11}+Q_{22}+i\left(Q_{12}-Q_{21}\right), \\
& W_{12}+W_{22}=Q_{11}-Q_{22}+i\left(Q_{12}+Q_{21}\right), \\
& W_{13}+W_{23}=-2\left(Q_{31}+i Q_{32}\right),
\end{aligned}
$$

and expressions for elements of $Q$ through the polar angles (5.8), we obtain from (5.39) the following explicit relation for the rotation coef cients through the reference frame rotation angles $\theta^{\prime}, \varphi^{\prime}$ and $\chi$ :

$$
\begin{aligned}
T_{n-1}^{\nu, m+1} & =\frac{e^{i \chi}}{b_{n}^{m}}\left\{\frac{1}{2}\left[b_{n}^{-\nu-1} e^{i \varphi^{\prime}}\left(1-\cos \theta^{\prime}\right) T_{n}^{\nu+1, m}-b_{n}^{\nu-1}\left(1+\cos \theta^{\prime}\right) e^{-i \varphi^{\prime}} T_{n}^{\nu-1, m}\right]-a_{n-1}^{\nu} \sin \theta^{\prime} T_{n}^{\nu m}\right\}, \\
n & =2,3, \ldots, \quad \nu=-n+1, \ldots, n-1, \quad m=0, \ldots, n-2,
\end{aligned}
$$

which enables computation of all $T_{n}^{\nu m}$ for positive $m$. This requires $O\left(N_{t}^{3}\right)$ operations for rotating a multipole series truncated at $n=N_{t}$ (i.e., for $O\left(N_{t}^{2}\right)$ coef cients).

Note also that recurrence relation (5.54) enables computation of the complex-valued functions $T_{n}^{\nu m}$. The computational procedure can be simpli ed if we use the factorization (5.19) and then rewrite (5.54) for the real-valued functions $H_{n}^{\nu m}\left(\theta^{\prime}\right)$ :

$$
\begin{aligned}
H_{n-1}^{\nu, m+1} & =\frac{1}{b_{n}^{m}}\left\{\frac{1}{2}\left[b_{n}^{-\nu-1}\left(1-\cos \theta^{\prime}\right) H_{n}^{\nu+1, m}-b_{n}^{\nu-1}\left(1+\cos \theta^{\prime}\right) H_{n}^{\nu-1, m}\right]-a_{n-1}^{\nu} \sin \theta^{\prime} H_{n}^{\nu m}\right\}, \\
n & =2,3, \ldots, \quad \nu=-n+1, \ldots, n-1, \quad m=0, \ldots, n-2,
\end{aligned}
$$

This process starts with initial value (5.48).

6. Rotation-Translation Operation. As is clear from the above theorem, the computation of the coaxial coef cients can be performed in $O\left(N_{t}^{3}\right)$ operations as opposed to $O\left(N_{t}^{4}\right)$ operations required for the general case. To take advantage of this fact for the general case we will consider the rotation of the coordinate system with rotation angles $\left(\Theta_{p q}, \Phi_{p q}, \chi_{p q}\right)$ to make the axis $\mathbf{i}_{z}$ directed from point $p$ to point $q$ and then apply the theory for coaxial coef cients. Such a rotation occurs in the plane determined by vectors $\mathbf{i}_{z}$ and $\mathbf{i}_{\bar{z}}=\mathbf{r}_{p q}^{\prime} /\left|\mathbf{r}_{p q}^{\prime}\right|$, and therefore:

$$
\cos \theta_{p q}=\mathbf{i}_{z} \cdot \mathbf{i}_{\bar{z}}=\frac{\mathbf{i}_{z} \cdot \mathbf{r}_{p q}^{\prime}}{\left|\mathbf{r}_{p q}^{\prime}\right|}=\frac{z_{q}^{\prime}-z_{p}^{\prime}}{r_{p q}^{\prime}}
$$

where $z_{q}^{\prime}$ and $z_{p}^{\prime}$ are $z$-coordinates of points $q$ and $p$ in the original coordinate system.

Equations (2.26) can be rewritten as

$$
E_{n}^{m}\left(\mathbf{r}_{p}\right)=\sum_{\nu=-n}^{n} T_{n}^{\nu m}\left(Q_{p q}^{\prime}\right) E_{n}^{\nu}\left(\widehat{\mathbf{r}}_{p}\right), \quad\left|\widehat{\mathbf{r}}_{p}\right|=\left|\mathbf{r}_{p}\right|, \quad E=S, R
$$

where $\widehat{\mathbf{r}}_{p}$ is the radius-vector of the point in the rotated coordinate system, and the rotation matrix $Q_{p q}^{\prime}\left(\Theta_{p q}, \Phi_{p q}, \chi_{p q}\right)$ is provided by (5.10).

The functions $E_{n}^{\nu}\left(\widehat{\mathbf{r}}_{p}\right)$ then can be translated/reexpanded near the reexpansion point $q$ according to (4.75)-(4.77):

$$
E_{n}^{\nu}\left(\widehat{\mathbf{r}}_{p}\right)=\sum_{l=|\nu|}^{\infty}(E \mid F)_{l n}^{\nu}\left(r_{p q}^{\prime}\right) F_{l}^{\nu}\left(\widehat{\mathbf{r}}_{q}\right), \quad F, E=S, R,
$$


where $\widehat{\mathbf{r}}_{q}$ is the radius vector centered at point $q$ in the rotated coordinate system. To return to the initial coordinates we rotate the coordinates back, so we perform rotation of coordinate system, speci ed by the rotation matrix $\left[Q_{p q}^{\prime}\right]^{T}=$ $Q_{p q}^{\prime-1}$ :

$$
F_{l}^{\nu}\left(\widehat{\mathbf{r}}_{q}\right)=\sum_{s=-l}^{l} T_{l}^{s \nu}\left(Q_{p q}^{\prime-1}\right) F_{l}^{s}\left(\mathbf{r}_{q}\right), \quad\left|\widehat{\mathbf{r}}_{q}\right|=\left|\mathbf{r}_{q}\right|, \quad E=S, R .
$$

Combining (6.2) and (6.4) we obtain:

$$
E_{n}^{m}\left(\mathbf{r}_{p}\right)=\sum_{\nu=-n}^{n} \sum_{l=|\nu|}^{\infty} \sum_{s=-l}^{l} T_{l}^{s \nu}\left(Q_{p q}^{\prime-1}\right) T_{n}^{\nu m}\left(Q_{p q}^{\prime}\right)(E \mid F)_{l n}^{\nu}\left(r_{p q}^{\prime}\right) F_{l}^{s}\left(\mathbf{r}_{q}\right), \quad E=S, R .
$$

Changing the order of summation of $l$ and $\nu$, we obtain

$$
E_{n}^{m}\left(\mathbf{r}_{p}\right)=\sum_{l=0}^{\infty} \sum_{s=-l}^{l} \sum_{\nu=-\min (l, n)}^{\min (l, n)} T_{l}^{s \nu}\left(Q_{p q}^{\prime-1}\right) T_{n}^{\nu m}\left(Q_{p q}^{\prime}\right)(E \mid F)_{l n}^{\nu}\left(r_{p q}^{\prime}\right) F_{l}^{s}\left(\mathbf{r}_{q}\right), \quad E=S, R . .
$$

On the other hand we have the representation (4.24). Comparing it with (6.6) and taking into account the fact that the elementary solutions $F_{l}^{s}\left(\mathbf{r}_{q}\right)$ are independent and orthogonal on a sphere with the center at the point $q$, we obtain:

$$
(E \mid F)_{l n}^{s m}\left(\mathbf{r}_{p q}^{\prime}\right)=\sum_{\nu=-\min (l, n)}^{\min (l, n)}(E \mid F)_{l n}^{\nu}\left(r_{p q}^{\prime}\right) T_{l}^{s \nu}\left(Q_{p q}^{\prime-1}\right) T_{n}^{\nu m}\left(Q_{p q}^{\prime}\right), \quad E=S, R .
$$

Note that according (5.9) and (5.19) we have

$$
\begin{aligned}
& T_{n}^{\nu m}\left(Q_{p q}^{\prime}\right)=T_{n}^{\nu m}\left(\Theta_{p q}, \Phi_{p q}, \chi_{p q}\right)=e^{i m \chi_{p q}} e^{-i \nu \Phi_{p q}} H_{n}^{\nu m}\left(\Theta_{p q}\right) \\
& T_{l}^{s \nu}\left(Q_{p q}^{\prime-1}\right)=T_{l}^{s \nu}\left(\Theta_{p q}, \Phi_{p q}, \chi_{p q}\right)=e^{-i s \chi_{p q}} e^{i \nu \Phi_{p q}} H_{l}^{s \nu}\left(\Theta_{p q}\right) .
\end{aligned}
$$

We also note that angles of rotation $\Theta_{p q}$ and $\chi_{p q}$, as de ned in (5.7), are nothing but the spherical polar angles $\theta_{p q}^{\prime}$ and $\varphi_{p q}^{\prime}$ of the vector $\mathbf{r}_{p q}^{\prime}=\left(r_{p q}^{\prime} \cos \varphi_{p q}^{\prime} \sin \theta_{p q}^{\prime}, r_{p q}^{\prime} \sin \varphi_{p q}^{\prime} \sin \theta_{p q}^{\prime}, r_{p q}^{\prime} \cos \theta_{p q}^{\prime}\right)$ in the original reference frame (in the rotated reference frame they are $\left.\widehat{\mathbf{r}}_{p q}^{\prime}=\left(0,0, r_{p q}^{\prime}\right)\right)$. Thus, the product of the two functions (6.8) depends only on these angles and can be expressed as

$$
\mathbb{T}_{l n}^{s \nu m}\left(\theta_{p q}^{\prime}, \varphi_{p q}^{\prime}\right)=T_{l}^{s \nu}\left(Q_{p q}^{\prime-1}\right) T_{n}^{\nu m}\left(Q_{p q}^{\prime}\right)=e^{i(m-s) \varphi_{p q}^{\prime}} H_{l}^{s \nu}\left(\theta_{p q}^{\prime}\right) H_{n}^{\nu m}\left(\theta_{p q}^{\prime}\right)
$$

and form (6.7) is a separation of the angular and distance variables for the translation reexpansion coef cients. Note that since rotation and inverse rotation preserve the vector, we have:

$$
\sum_{\nu=-\min (l, n)}^{\min (l, n)} \mathbb{T}_{l n}^{s \nu m}=\delta_{l n} \delta_{s m} .
$$

6.1. Relation Between Rotation and Translation Reexpansion Coef cients. The structure of the coef cients $\mathbb{T}_{l n}^{s \nu m}\left(\theta_{p q}^{\prime}, \varphi_{p q}^{\prime}\right)$ can be found using (4.8) for the $(S \mid R)$-coef cients, or (4.18) for the $(R \mid R)$-coef cients. Substituting (4.8) for $(S \mid R)_{l n}^{s m}\left(\mathbf{r}_{p q}^{\prime}\right)$ and $(S \mid R)_{l n}^{\nu}\left(r_{p q}^{\prime}\right)=\left.(S \mid R)_{l n}^{\nu \nu}\left(\widehat{\mathbf{r}}_{p q}^{\prime}\right)\right|_{\hat{\theta}_{p q}^{\prime}=0}$ in (6.7) we obtain, using the de nition of the multipoles (2.14):

$$
\sum_{\alpha=0}^{\infty} h_{\alpha}\left(k r_{p q}^{\prime}\right) \sum_{\beta=-\alpha}^{\alpha}(s \mid r)_{\alpha l n}^{\beta s m} Y_{\alpha}^{\beta}\left(\theta_{p q}^{\prime}, \varphi_{p q}^{\prime}\right)=\sum_{\alpha=0}^{\infty} h_{\alpha}\left(k r_{p q}^{\prime}\right) \sum_{\beta=-\alpha \nu=-\min (l, n)}^{\alpha} \sum_{\beta}^{\min (l, n)}(s \mid r)_{\alpha l n}^{\beta \nu \nu} \mathbb{T}_{l n}^{s \nu m}\left(\theta_{p q}^{\prime}, \varphi_{p q}^{\prime}\right) Y_{\alpha}^{\beta}(0,0) .
$$

Since the functions $h_{\alpha}\left(k r_{p q}^{\prime}\right)$ at $\alpha=0,1, \ldots$ are linearly independent, each term in the sum over $\alpha$ in the left hand side of this equation must be equal to the corresponding term on the right hand side. We also notice that due to (4.14) 
only one term on each side of equation (6.11) represents the sum over $\beta$. So, dropping the prime and the subscript near the spherical angles we have

$$
(s \mid r)_{\alpha l n}^{m-s, s m} Y_{\alpha}^{m-s}(\theta, \varphi)=\sqrt{\frac{2 \alpha+1}{4 \pi}} \sum_{\nu=-\min (l, n)}^{\min (l, n)}(s \mid r)_{\alpha l n}^{0 \nu \nu} \mathbb{T}_{l n}^{s \nu m}(\theta, \varphi), \quad \alpha, l, n=0,1, \ldots
$$

This relation is very general since it holds at arbitrary $\alpha, l, n, m$, and $s$. In the particular case $s=0$, substituting (6.9) and (5.55) here, we obtain:

$$
(s \mid r)_{\alpha l n}^{m 0 m} Y_{\alpha}^{m}(\theta, \varphi)=\sqrt{\frac{2 \alpha+1}{2 l+1}} \sum_{\nu=-\min (l, n)}^{\min (l, n)}(s \mid r)_{\alpha l n}^{0 \nu \nu} e^{i(m-\nu) \varphi} H_{n}^{\nu m}(\theta) Y_{l}^{\nu}(\theta, \varphi) .
$$

For $m=0$ this yields

$$
(s \mid r)_{\alpha l n}^{000} Y_{\alpha}^{0}(\theta, \varphi)=\sqrt{\frac{4 \pi(2 \alpha+1)}{(2 l+1)(2 n+1)}} \sum_{\nu=-\min (l, n)}^{\min (l, n)}(s \mid r)_{\alpha l n}^{0 \nu \nu} Y_{n}^{-\nu}(\theta, \varphi) Y_{l}^{\nu}(\theta, \varphi)
$$

while for $n=m$ using the expression for $H_{m}^{\nu m}(\theta)$ following from (5.20) we obtain

$$
(s \mid r)_{\alpha l m}^{m 0 m} Y_{\alpha}^{m}(\theta, \varphi)=\sum_{\nu=-\min (l, m)}^{\min (l, m)} \epsilon_{m} \epsilon_{\nu}\left[\frac{(2 \alpha+1)(2 m) !}{(2 l+1)(m+\nu) !(m-\nu) !}\right]^{1 / 2}(s \mid r)_{\alpha l m}^{0 \nu \nu} \cos ^{m+\nu} \frac{\theta}{2}\left[e^{i \varphi} \sin \frac{\theta}{2}\right]^{m-\nu} Y_{l}^{\nu}(\theta, \varphi) .
$$

These formulae provide relations between spherical harmonics of different order and degree. We also note that $\mathbb{T}_{l n}^{s \nu m}(\theta, \varphi)$ are surface functions that can be expanded in terms of spherical harmonics:

$$
\mathbb{T}_{l n}^{s \nu m}(\theta, \varphi)=\sum_{\gamma=|m-s|}^{\infty} t_{l \gamma n}^{s \nu m} Y_{\gamma}^{m-s}(\theta, \varphi)
$$

where the numerical rotation coef cients $t_{\gamma l n}^{s \nu m}$ can be related to the translation reexpansion coef cients $(s \mid r)_{\gamma l m}^{s \nu m}$ (or to Wigner 3- $j$ and other symbols, see (4.13) and above).

6.2. Complexity of Rotation-Coaxial Translation Decomposition. It must be noted that the representation (6.6) and the above expressions for $\mathbb{T}_{l n}^{s \nu m}(\theta, \varphi)$ in the form of series are conceptual. In practice computation of the translation reexpansion coef cients $(E \mid F)_{l n}^{s m}\left(\mathbf{r}_{p q}^{\prime}\right)$ would be performed by a composition of successive products (thereby avoiding expensive matrix-matrix products). The computational advantage of translation decomposition is that one can perform the rotation operation (which requires $O\left(N_{t}^{3}\right)$ operations), and then the coaxial translation, which also can be performed for $O\left(N_{t}^{3}\right)$ operations, and then (if needed), rotation that can again be made for $O\left(N_{t}^{3}\right)$ operations. So the total number of operations for such a procedure is $O\left(N_{t}^{3}\right)$ opposed to $O\left(N_{t}^{4}\right)$ operations required for a general translation.

\section{Summary and Conclusions.}

7.1. Summary of Basic Recursions. As the paper dealt with many recurrence relations, the tables below provide a summary of the main results. In the columns, we list the indices of the translation and rotation coef cients, or their shifts, that are involved in the recursions listed in the columns to the left.

Translation coef cients 
Rotation Coef cients

\begin{tabular}{|l|l|l|l|l|}
\hline Formula & $n$ & $l$ & $m$ & $s$ \\
\hline $\mathbf{( 4 . 2 6 )}$ & $-1,0,1$ & $-1,0,1$ & 0 & 0 \\
\hline $\mathbf{( 4 . 3 0 )}$ & $-1,0,1$ & $-1,0,1$ & 0,1 & $-1,0$ \\
\hline $\mathbf{( 4 . 3 4 )}$ & $-1,0,1$ & $-1,0,1$ & $-1,0$ & 0,1 \\
\hline$(4.27)$ & $=|m|$ & $-1,0,1$ & $0, \pm 1$ & 0 \\
\hline$(4.28)$ & $-1,0,1$ & $=|s|$ & 0 & $0, \pm 1$ \\
\hline$(4.29)$ & $=|m|$ & $=|s|$ & $0, \pm 1$ & $0, \pm 1$ \\
\hline$(4.31)$ & $=m$ & $-1,0,1$ & $0,1(\geqslant 0)$ & $-1,0$ \\
\hline$(4.32)$ & $-1,0,1$ & $=-s$ & 0,1 & $-1,0(\leqslant 0)$ \\
\hline$(4.33)$ & $=m$ & $=-s$ & $0,1(\geqslant 0)$ & $-1,0(\leqslant 0)$ \\
\hline$(4.35)$ & $=-m$ & $-1,0,1$ & $-1,0(\leqslant 0)$ & 0,1 \\
\hline$(4.36)$ & $-1,0,1$ & $=s$ & $-1,0$ & $0,1(\geqslant 0)$ \\
\hline$(4.37)$ & $=-m$ & $=s$ & $-1,0(\leqslant 0)$ & $0,1(\geqslant 0)$ \\
\hline$(4.80)$ & $-1,0,1$ & $-1,0,1$ & 0,1 & $=m$ \\
\hline$(4.84)$ & $=m$ & $-1,0,1$ & $0,1(\geqslant 0)$ & $=m$ \\
\hline
\end{tabular}

\begin{tabular}{|l|l|l|l|}
\hline Formula & $n$ & $m$ & $\nu$ \\
\hline$(5.24)$ & 0,1 & $-1,0,1$ & $-1,0,1$ \\
\hline$(5.25)$ & 0,1 & $-1,0,1$ & $-1,0,1$ \\
\hline$(5.26)$ & 0,1 & 0 & $-1,0,1$ \\
\hline$(5.36)$ & $-1,0$ & $-1,0,1$ & $-1,0,1$ \\
\hline$(5.37)$ & $-1,0$ & $-1,0,1$ & $-1,0,1$ \\
\hline$(5.38)$ & $-1,0$ & 0 & $-1,0,1$ \\
\hline$(5.55)$ & $-1,0$ & 0,1 & $-1,0,1$ \\
\hline
\end{tabular}

7.2. Conclusions. We have presented a method for fast computation of the multipole translation and rotation coef cients for the 3-D Helmholtz equation using recurrence relations, which are derived. This method enables computation of the full matrix of translation coef cients for a multipole expansion truncated at $N_{t}$ terms in degree (the total number of expansion coef cients is $\left.O\left(N_{t}^{2}\right)\right)$ using $O\left(N_{t}^{4}\right)$ operations, as opposed to $O\left(N_{t}^{5}\right)$ operations required for computations using the Wigner or Clebsch-Gordan summations. We provided an $O\left(N_{t}^{4}\right)$ algorithm for translation of multipole expansions and proved recurrence theorems for translation coef cients. These theorems also were checked numerically by comparing the exact values of multipoles and the values computed using multipole reexpansions. Using rotation-coaxial translation decomposition of the translation operators, the set of $N_{t}^{2}$ expansion coef cients for a solution of the Helmholtz equation can be computed in $O\left(N_{t}^{3}\right)$ operations, if coaxial coef cients are used. Since we provide explicit relations requiring two or three multiplications/additions, the constants multiplying the order symbols for all our algorithms are small.

Acknowledgements. We would like to gratefully acknowledge the support of NSF awards 0086075 and 0219681. We also thank the reviewers whose comments improvement the initial version of this manuscript.

\section{REFERENCES}

[1] J.J. Dongarra and F. Sullivan, "The top 10 algorithms." Computing in Science \& Engineering, 2, 22-23, 2000.

[2] N.A. Gumerov and R. Duraiswami, "Fast, exact, and stable computation of multipole translation and rotation coef cients for the 3-D Helmholtz equation," University of Maryland, Institute for Advanced Computer Studies, UMIACS Technical Report TR 2001-44, 2001 (available online).

[3] P. Moon and D.E. Spencer, Field Theory Handbook, Springer-Verlag, 3rd printing, 1988.

[4] L. Greengard, The Rapid Evaluation of Potential Fields in Particle Systems. Cambridge, MA: MIT Press, 1988.

[5] P.M. Morse and H. Feshbach, Methods of Theoretical Physics -I, McGraw-Hill, 1953.

[6] M. Abramowitz and I.A. Stegun. Handbook of Mathematical Functions, National Bureau of Standards, Wash., D.C., 1964.

[7] R. Coifman, V. Rokhlin, and S. Wandzura, "The fast multipole method: a pedestrian prescription." IEEE Antennas and Propagation Mag., 3(35), 7-12, 1993.

[8] M.A. Epton and B. Dembart, "Multipole translation theory for the three-dimensional Laplace and Helmholtz equations." SIAM J. Scienti c Computing, 4(16), 865-897, 1995.

[9] V. Rokhlin, "Diagonal form of translation operators for the Helmholtz equation in three dimensions." Applied and Computational Harmonic Analysis, 1(1), 82-93, 1993.

[10] J.M. Song and W.C. Chew, "Multilevel fast-multipole algorithm for solving combined eld integral equations of electromagnetic scattering." Microwave Opt. Technol. Lett., 10, 14-19, 1995. 
[11] J. Rahola, "Experiments on iterative methods and the fast multipole method in electromagnetic scattering." CERFACS Technical Report TR/PA/98/49, CERFACS, Toulous, 1998.

[12] L. Greengard and V. Rokhlin, “A fast algorithm for particle simulations.” J. Comput. Phys., 73, 325-348, 1987.

[13] L. Greengard, J. Huang, V. Rokhlin, and S. Wandzura, "Accelerating fast multipole methods for the Helmholtz equation at low frequencies," IEEE Comp. Sci. Engng, 5(3), 32-38,1998.

[14] S. Koc, J. Song, and W.C. Chew, "Error analysis for the numerical evaluation of the diagonal forms of the scalar spherical addition theorem." SIAM J. Numer. Anal., 36(3), 1999, pp. 906-921.

[15] S. Stein, "Addition theorems for spherical wave functions." Quart. Appl. Math., 19, 15-24, 1961.

[16] X. Sun and N.P. Pitsianis, "A matrix version of the fast multipole method," SIAM Review, 43, 289-300, 2001.

[17] E. Wigner, Gruppentheorie und ihre Anwendung auf die Quanten-mechanik der Atomspektren, Vieweg, Wiesbaden, Germany, 1931.

[18] J. Ivanic and K. Ruedenberg, "Rotation matrices for real spherical harmonics. Direct determination by recursion," J. Chem. Phys.. 100, 6342-6347, 1996; (errata 102, 9099-9100, 1998).

[19] C.H. Choi, J. Ivanic, M. S. Gordon, and K. Ruedenberg, "Rapid and stable determination of rotation matrices between spherical harmonics by direct recursion," J. Chem. Phys. ,111, 8825-8831, 1999.

[20] H. Goldstein, Classical Mechanics, 2nd ed., Narosa, 1980.

[21] E. Darve. "The fast multipole method: numerical implementation", J. Comput. Phys., 160(1), 195-240, 2000.

[22] W.C. Chew, "Recurrence realtions for three-dimensional scalar and addition theorem," J. Electromagn. Waves Appl. 6(2), 133-142, 1992.

[23] W.C. Chew, J. M. Jin, E. Michielssen, and J. Song, Fast and Ef cient Algorithms in Computational Electromagnetics, Artech House, 2001.

[24] N.A. Gumerov and R. Duraiswami, "Computation of scattering from $N$ spheres using multipole reexpansion," J. Acoust. Soc. Am. 112(6), 2688-2701, 2002.

[25] N.A. Gumerov, R. Duraiswami, and E.A. Borovikov, "Data structures, optimal choice of parameters, and complexity results for generalized multilevel fast multipole methods in d dimensions" University of Maryland, Institute for Advanced Computer Studies, UMIACS Technical Report TR 2003-28, 2003 (available online).

[26] N.A. Gumerov and R. Duraiswami, "Acoustical scattering from $N$ spheres using a multilevel fast multipole method," J. Acoust. Soc. Am.,113 (4) pt. 2, 2334, 2003.

[27] M.F. Gyure and M.A. Stalzer, “A Prescription for the Multilevel Helmholtz FMM", IEEE Computational Science and Engineering, Vol. 5(3), pp. 39-47, 1998. 